\title{
Características de la producción científica de instituciones de Puebla indexada en la Web of Science
}

\author{
Alfonso Felipe Díaz-Cárdenas \\ María del Rayo Sankey-García \\ Alfonso Díaz-Furlong* \\ Héctor Adrián Díaz-Furlong** \\ Reyna Xoxocotzi-Aguilar*** \\ José JaimeVázquez-López \\ Cynthia Apam-García ${ }^{\star \star \star \star}$
}

Artículo recibido:

3 de agosto de 2015

Artículo aceptado:

2 de mayo de 2016
RESUMEN

En este artículo describimos el ámbito de la investigación en el estado de Puebla, México, cuyos resultados han sido publicados en revistas indizadas en la Web of Science (WoS). El corpus examinado fue recopilado mediante el procedimiento de búsqueda con la palabra "puebla" en los campos organización y dirección, restringido al periodo 2008-2012. Como se desprende de

Facultad de Psicología, Universidad Autónoma de Puebla; Universidad Autónoma de Puebla, México. Department of Physics, McGill University, Montreal, Canadá diazcard@yahoo.com, ryathome@hotmail.com, alfonso.furlong@correo.buap.mx. * Colegio de Arte Digital, Escuela de Artes Plásticas y Audiovisuales, Universidad Autónoma de Puebla, México. hector.diazfurlong@correo.buap.mx.

*** Escuela de Ciencias Físico Matemáticas, Universidad Autónoma de Puebla, México.xoxo_reyna@yahoo.com.mx.

**** Coordinación General de Proyectos BUAP, México. jaime.vazquez@correo.buap.mx cynthia.apam@correo.buap.mx.

INVESTIGACIÓN BIBLIOTECOLÓGICA, Número Especial de Bibliometría, 2017, México, ISSN: 2448-8321, pp. 43-77 
los datos obtenidos la Universidad Autónoma de Puebla y el Instituto Nacional de Astrofísica, Óptica y Electrónica son los organismos más productivos del estado de Puebla. Nos centramos en los 102 artículos más citados del periodo y observamos que la producción científica con participación de instituciones poblanas se concentra en las ciencias físicas y biomédicas. Esta producción, mayoritariamente, proviene de colaboraciones de carácter internacional y las publicaciones más citadas presentan al menos tres autores; mientras que los textos de responsabilidad individual tienen poco impacto, de acuerdo con el número de citas registradas. Asimismo, estudiamos las diferencias por áreas científicas en cuanto a producción e impacto en términos de citas.

Palabras clave: Citas; Impacto; Temas relevantes de investigación; Autoría múltiple; Indicadores bibliométricos; Desempeño de la investigación.

\section{Characteristics of the scientific production of Puebla} institutions indexed in the Web of Science

Alfonso Felipe Díaz-Cárdenas, María del Rayo SankeyGarcía, Alfonso Díaz-Furlong, Héctor Adrián DíazFurlong, Reyna Xoxocotzi-Aguilar, José Jaime VázquezLópez and Cynthia Apam-García

\section{Abstract}

This article describes the scope and characteristics of the research developed in the state of Puebla, Mexico, whose results have been published in journals indexed in the Web of Science. The analyzed corpus was compiled by the search procedure with the word "puebla" in the organization and address fields, restricted to the period 20082012. We observed from the data obtained that the Universidad Autónoma de Puebla and the Instituto Nacional de Astrofísica, Óptica y Electrónica are the most productive institutions of Puebla. Our focus was on the 102 most cited articles of the period. Scientific production of Pue- 
CARACTERÍSTICAS DE LA PRODUCCIÓN CIENTÍFICA DE INSTITUCIONES DE PUEBLA...

sciences and this production derives mostly from international collaborations. The multiauthor articles (at least three authors) are the most cited publications, while the individual research papers have little impact according to their number of citations. Finally, we analyze the differences in impact factor across scientific fields.

Keywords: Citations; Most-cited; Science trends topics; Multiple authorship; Bibliometric indicators; Research performance.

\section{INTRODUCCIÓN}

E n este artículo analizamos el ámbito de la investigación en el estado de Puebla, México, cuyos resultados han sido publicados en revistas indizadas en la Web of Science (WoS), que incluye el Science Citation Index Expanded (SCIE), Social Sciences Citation Index (SSCI) y Arts \& Humanities Citation Index (A\&HCI). La elección de esta base de datos, de resultados de indagación científica, obedece a su relevancia en el criterio que rige la clasificación de universidades y centros de investigación en cuanto a su productividad. Ciertamente, la categorización de estas instituciones en el ámbito internacional toma, entre otras, a la WoS como indicador del impacto de las publicaciones, a través del número de citas a sus trabajos editados. Una de las más reconocidas, por ejemplo, la llamada clasificación de Shanghai (The Academic Ranking of World Universities) se basa en los documentos indizados en SCIE y SSCI.

El corpus examinado 5 se recopiló mediante el procedimiento de búsqueda usual entre la comunidad científica y escolar: elegimos la palabra "puebla" en los campos organización y dirección como criterio de sondeo entre la información que proporciona este sistema y se restringió al periodo 2008-2012. En una búsqueda inicial, fueron descargados ${ }^{6}$ datos preliminares el día 21 de agosto de 2012. Por supuesto, seleccionamos sólo los documentos que fueran autoría de investigadores cuya dirección postal o institucional correspondiera a la comunidad elegida. Básicamente, el análisis de este documento se enfoca en los datos obtenidos en febrero de 2013. Además, previo a la redacción final del texto, realizamos una actualización el día 7 de mayo de 2013.

5 Un conjunto de datos incluidos aquí se tomaron de la Web of Science, ${ }^{\circledR}$ preparados por Thomson Reuters Scientific, Inc. (Thomson®), Filadelfia, Pensilvania, Estados Unidos: (C) Copyright Thomson Reuters ® 2012, 2013. Todos los derechos reservados.

6 En un artículo enmendado, la Real Academia Española en una de sus acepciones define la palabra descargar como: Inform. Transferir información desde un sistema electrónico a otro (RAE, 2014: 745). 


\section{DistRIBUCión DE CITAS}

Nuestra primera labor es poner de relieve la frecuencia de citas a los artículos contenidos en el corpus. ${ }^{7}$ Describiremos, después, las características de los documentos que ocupan las primeras posiciones en cuanto al número de citas y, finalmente, a partir de esta tarea, nos detendremos en una reflexión sobre la investigación científica en nuestra entidad.

La WoS, en el periodo elegido, registra 3,585 artículos en revistas indizadas en aquélla. El promedio total del periodo es de 4.28 citas por escrito. De éstos, como se observa en el Cuadro 1, el 52.08\% no ha sido citado -por razones de espacio, los Cuadros 1 y 2 aparecen seccionados y emparejados-. Esto no es insólito, ya que, de acuerdo con la regla 80/20, en muchas revistas el $80 \%$ de las citas son obtenidas por el 20\% de sus artículos (Dorta y Dorta, 2010). En lo sucesivo, nos centraremos en los documentos que ocupan los primeros noventa y cinco lugares en cuanto al número de citas. De estos textos, uno cuenta con 524 citas; cuatro recibieron más de 200; once alcanzan más de las 100 y los restantes documentos registran entre 30 a 95 citas. Estos ciento dos textos representan el $2.2 \%$ del total del corpus (Cuadro 1) y son el eje de la subsecuente discusión.

\begin{tabular}{|c|c|c|c|c|c|c|c|c|c|c|c|}
\hline 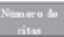 & Frectemia & 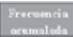 & Precostate & Nimaresto & Fresuenta & Frecamentit & Parcientis & Nomedo & Frectentis & 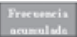 & Porrent is: \\
\hline 524 & 1 & 1 & 003 & 67 & 1 & 5 & 0.63 & 3 & 3 & 107 & 298 \\
\hline $22 \mathrm{a}$ & 1 & 2 & 003 & 63 & 2 & s4 & 0.06 & 28 & $t$ & 115 & 221 \\
\hline 273 & 1 & 3 & 0.03 & 50 & 1 & 35 & a.13 & 27 & 7 & 122 & 340 \\
\hline 223 & 1 & . & 003 & $s 8$ & 1 & 30 & 003 & $2 s$ & 2 & 124 & 346 \\
\hline 217 & 1 & 1 & 003 & 37 & 1 & 3 & 0.03 & r & 3 & 129 & 3.60 \\
\hline 142 & i & 6 & 0.03 & 56 & 2 & 39 & 0.06 & ${ }_{24}$ & s & 134 & 374 \\
\hline 18 & $i$ & ; & 003 & 35 & 2 & 41 & 0.08 & 2) & 6 & 140 & 391 \\
\hline 138 & 1 & 8 & 003 & $\$ 4$ & 1 & 42 & 0.03 & 22 & 10 & 150 & 4.18 \\
\hline 130 & 1 & 9 & 003 & 53 & 1 & 43 & 0.03 & 21 & 13 & 163 & 455 \\
\hline 122 & $i$ & "1" & 003 & 32 & $i$ & t4 & 0.03 & 20 & " & 169 & 471 \\
\hline 115 & $i$ & ॥ & 003 & st & $i$ & 45 & 0.03 & 13 & 12 & m & 505 \\
\hline 114 & 1 & 12 & 003 & 49 & 3 & 43 & 0.68 & 13 & 14 & 135 & 5.44 \\
\hline 111 & 1 & 13 & 0.03 & 48 & 2 & so & 0.06 & 17 & 14 & 209 & 5.83 \\
\hline 100 & 1 & 14 & 003 & 47 & 3 & s3 & 0.63 & 15 & 13 & 222 & 6.19 \\
\hline 107 & 1 & is & 003 & 45 & 2 & $s s$ & 0.06 & is & 14 & 236 & 658 \\
\hline 105 & 1 & "s & 003 & 44 & 2 & 57 & 0.56 & is & Is & $\mathrm{si}_{1}$ & 200 \\
\hline 93 & $i$ & $a$ & 003 & 43 & $i$ & $s$ & 0,63 & B & (x) & 231 & 284 \\
\hline 92 & 1 & "1 & 003 & 42 & 1 & $\$ 9$ & 0.03 & 12 & a & 392 & 842 \\
\hline 93 & $i$ & is & 003 & $"$ & 2 & 61 & 0.68 & " & 41 & w3 & 957 \\
\hline 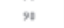 & $i$ & $a$ & 003 & 90 & 3 & CA & 0.06 & a & $\bar{x}$ & ssi & 1203 \\
\hline 83 & 1 & at & 003 & 39 & 1 & 65 & 0.03 & , & 41 & 422 & แ. $\pi$ \\
\hline n: & 1 & 22 & 003 & 38 & 2 & 67 & 0.06 & $*$ & $s$ & 479 & 1236 \\
\hline t: & 2 & 24 & 0.06 & 37 & 2 & $\theta$ & 0.00 & , & $\$$ & 332 & 14.54 \\
\hline " & $i$ & 25 & 003 & 36 & 2 & $n$ & 0.66 & 6 & $n$ & coss & 16.83 \\
\hline$"$ & 1 & as & 003 & is & 6 & $\pi$ & 0.17 & 3 & s & sto & 1822 \\
\hline$n$ & i & 27 & 003 & ${ }_{34}$ & 3 & 42 & 0.14 & 4 & 140 & $m$ & 28.12 \\
\hline$n$ & $i$ & it & 003 & 3 & 4 & is & 0.11 & 3 & 186 & 985 & 20.45 \\
\hline$n$ & 1 & 23 & 0003 & 12 & $s$ & 9 & 0.14 & 2 & 236 & 131 & 40.2 \\
\hline$n$ & 1 & $n$ & 003 & n & , & 94 & o.ts & 1 & $4 \pi$ & 1778 & 4792 \\
\hline 69 & 1 & x & 0.03 & 30 & 8 & 102 & 0.22 & 0 & 1867 & 3485 & 5203 \\
\hline
\end{tabular}

7 Cabe recordar que la tasa de citas varía considerablemente, de acuerdo con la disciplina que se analiza. Ciertamente, mientras que las citas alcanzan su máximo en pocos meses en las áreas médicas, biológicas o en las ciencias de la física, para que un documento atraiga un número importante de citas puede tomar más tiempo en las artes, las humanidades o en las ciencias sociales (Testa, 2003). 
CARACTERÍSTICAS DE LA PRODUCCIÓN CIENTÍFICA DE INSTITUCIONES DE PUEBLA...

El Cuadro 1 ordena las citas de forma descendente y presenta la frecuencia y el porcentaje acumulado. El incremento en el número de citas en los periodos registrados (agosto de 2012; febrero de 2013; mayo de 2013) se indica en el Cuadro 2.

Cuadro 2. Relación de los 102 documentos más citados

\begin{tabular}{|c|c|c|}
\hline Powition & Auther & Gits \\
\hline 01001 & 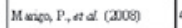 & 443224549 \\
\hline $03[\ln ] 06$ & 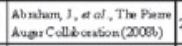 & $200024 / 20$ \\
\hline $02(020) 04$ & 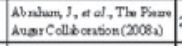 & 2002323 \\
\hline 0601010s & ALCE E ollib cention (2008) & 165020231 \\
\hline $74[050) 03$ & CMS wllbortition (2012a) & $28 / 21>2 * 4$ \\
\hline овш⿻ & 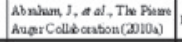 & 111242163 \\
\hline ormeses & A._.CE & कo: \\
\hline ospajun & $\begin{array}{l}\text { Cutementh, RA, etal } \\
(8008)\end{array}$ & 1170.28135 \\
\hline 1200909 & 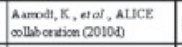 & 85130148 \\
\hline 21[10110 & Duriky.J. et al (2011) & 651222145 \\
\hline $10[11] 12$ & 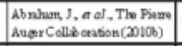 & 90116130 \\
\hline $08[122] 15$ & CMS malboration (2011) 15) & $96 / 114 / 15$ \\
\hline$-(13) \infty$ & CMS moldotoratican (2013) & anes \\
\hline 3ulaj 14 & 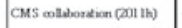 & 49009718 \\
\hline $13[2]$ & 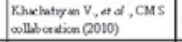 & 2010212 \\
\hline$\pi(1.17$ & Dowiin M J, a d (2009) & 991005105 \\
\hline 1601278 & Evas,S.enal (2010) & nesnos \\
\hline $14[18] 19$ & 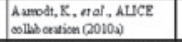 & 789297 \\
\hline $11[10322$ & 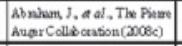 & soryss \\
\hline 35[2]15]15 & 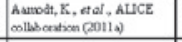 & 4า \\
\hline 16[2]134 & 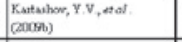 & 728085 \\
\hline $15[2] 2]$ & Seott, Ks, nal. (2008) & 758282 \\
\hline $25[2]]^{2}$ & 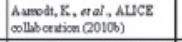 & 608189 \\
\hline $19[2] 25$ & 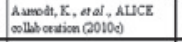 & 6\%:L84 \\
\hline $30[2] 252$ & 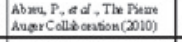 & s18093 \\
\hline$x \cos x=$ & CMS ollaboratican (2012A) & 122996 \\
\hline $18[2] 2]$ & 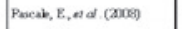 & 697880 \\
\hline $19[20029$ & $C_{\text {Qpp }}$,, $\mathrm{K}$, , efal. (2008) & 692072 \\
\hline $44[20] 28$ & 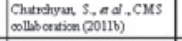 & 392179 \\
\hline 20502350 & Marden G. a d d. (2009) & 62707 \\
\hline $22(31) 30$ & 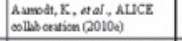 & 646571 \\
\hline $22[323] 3$ & 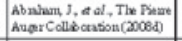 & 646767 \\
\hline $25[33] 36$ & 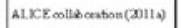 & so63ass \\
\hline$m \pi n n$ & 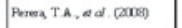 & 556263 \\
\hline
\end{tabular}

\begin{tabular}{|c|c|c|}
\hline Possicition & Auter & Cits \\
\hline 2803532 & CMS colles cration(J)(B) & 530267 \\
\hline 312040 & Vimo, M.P, enel. $(2009)$ & 500259 \\
\hline 202735 & Neorlla, M, a al. (2010) & 40,2254 \\
\hline 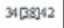 & CMS edliv cention (20106) & 4815657? \\
\hline $40[\sqrt{2}] 42$ & Fowlex, J.W, etal. (2010) & 41115657 \\
\hline 284ames & Dyes, s, etal. (rns) & sassoss \\
\hline 344046 & 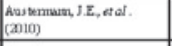 & acsos \\
\hline 204200 & Du,s,enel.(2011) & 30,4659 \\
\hline 31 (45)/48 & Pasuchon, G a e ol (2009) & 505254 \\
\hline 374046s & 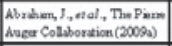 & 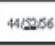 \\
\hline 6661.239 & Maniso, TA, eal. (xol1) & 3011150 \\
\hline 3040953 & Irvon R.J, et d. (2010) & 424249 \\
\hline 8140969 & 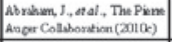 & $3020 \times 3$ \\
\hline 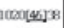 & CMS collibceston(2)120) & 1 198\% \\
\hline$x$ e[9]56 & Chrents, D L, ent. (2000) & 404848 \\
\hline 449955 & Lan,, , a al $(2009)$ & 994852 \\
\hline 40151235 & CMS eollibention(2011d) & 4142\%9 \\
\hline 48515] & Ambluxh, A, et al (2010) & замак? \\
\hline $2[51] \div 2$ & CMS collex cation(20110) & 274757 \\
\hline $40[5453$ & 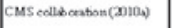 & Dusser \\
\hline 965451 & Sethal N, eal (2011) & 204551 \\
\hline 4250090 & (100es, F, nall (2008) & $40 \div 444$ \\
\hline \$85อక1 & ALICE collabontion (2011e) & 324451 \\
\hline $\operatorname{sins962}$ & 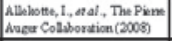 & 3043\%3 \\
\hline $4[52565$ & B waiblos, S, at al. (2000) & 304242 \\
\hline exखण 52 & CMS collis catoson(3011b) & 37414:43 \\
\hline SA ELIOB & Coblers IM, ed (2) & mo1/41 \\
\hline 5062265 & $\begin{array}{l}\text { Remivesuanfoun JC, enal. } \\
(2006)\end{array}$ & 354242 \\
\hline 5282870 & Konhl U., a d : (2005) & 344009 \\
\hline 5182599 & 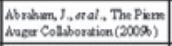 & 3a40us \\
\hline s489358 & Sthañ $Z$, a d al $(2009)$ & $30390 \% 6$ \\
\hline 6680667 & 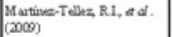 & 3000043 \\
\hline 8706065 & Millor, ps, ad (2010) & 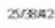 \\
\hline s[x:30 & 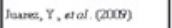 & xernas \\
\hline
\end{tabular}

\begin{tabular}{|c|c|c|}
\hline Punicion & Action & Cits \\
\hline $63(60368$ & CMS colleboantion(3) IC) & $31 / 32 / 41$ \\
\hline $66[20778$ & Bisgurl, H, a d al. (2008) & $3058 / 56$ \\
\hline sıcons & Hincls, A D, enal. (2010) & $25 S 6 / 37$ \\
\hline$s[272283$ & $G$ misuthy, $R, a$ al $(2008)$ & 3013/35 \\
\hline$\infty 6[22] 75$ & $\operatorname{seckin} V N$, et d $d(2010)$ & $3013 / 30$ \\
\hline $74[2218$ & Sect, KS, eal (xio) & $2025 / x$ \\
\hline 960275 & 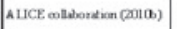 & $20[3 / 3$ \\
\hline 1000203 & AUCE wlatordion (20104) & $2020 / 25$ \\
\hline 100002250 & CM3 collubontion (20120) & $1 / 2539$ \\
\hline scasp & Parede, $\bar{\varepsilon}$, en al. $(2009)$ & 328456 \\
\hline $65[2887$ & Dye,, , etal $(2000)$ & $31 / 34 / 34$ \\
\hline $63[2397$ & Patarichon,,$+\& d .(008)$ & $31 / 34 / 34$ \\
\hline 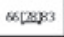 & $\begin{array}{l}\text { Rodrizues Zunth } G \text {, etal. } \\
\text { (amos) }\end{array}$ & 300435 \\
\hline 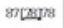 & Hoxtmam, IM, eal (ant) & $250 \sin x$ \\
\hline sexpes & Szemeit, $A$, e ol (2009) & $30 \mathrm{~m} / \mathrm{m}$ \\
\hline $74[20387$ & $\begin{array}{l}\text { Gúme-Almover, D, etal } \\
(2008)\end{array}$ & 2850254 \\
\hline 80000970 & seiv, T, a al, (2009) & $2602 y=$ \\
\hline 1278.3978 & CMS collebontion(201in & $19 / 33 / 56$ \\
\hline 7488794 & 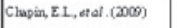 & 2020353 \\
\hline 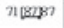 & Clapen, E1, etal (ADB) & $2020 / 54$ \\
\hline-18290 & CMS colldhontion (201 14) & 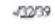 \\
\hline 12780787 & 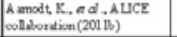 & $1920 / 54$ \\
\hline 5998787 & 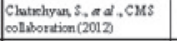 & $4 / 2234$ \\
\hline 71029295 & 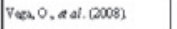 & $2912 / 39$ \\
\hline 7402292 & $\begin{array}{l}\text { Kantwhor, } Y . Y, \text { etal. } \\
(2009)\end{array}$ & $2031 / 33$ \\
\hline 17802500 & 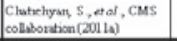 & $14[3 / 4 / 40$ \\
\hline $7 \log 2 \pi$ & 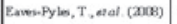 & $2 x+53$ \\
\hline 7902102 & 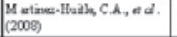 & $2 \pi 00 / 1$ \\
\hline 820525102 & Hech, G., nal $(2000)$ & 2600/1 \\
\hline 8702594 & Ahnuier G, en d. (2000) & 250050 \\
\hline $87 \cos 597$ & Wentefied, C.B, a al ( $(2000)$ & $25300 / 30$ \\
\hline $87(05) 102$ & $\begin{array}{l}\text { Awtemam, JE, ed. } \\
(2009)\end{array}$ & $2530 / 31$ \\
\hline x[x]108 & 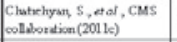 & $2 \pi x+30$ \\
\hline $96[6] 108$ & $L a, H B, \& d(200)$ & $24 / n \pi n$ \\
\hline
\end{tabular}


Los documentos (véanse las referencias) se enlistan en orden descendente, de acuerdo con el número de citas registrado en febrero de 2013. Se indica, en ambas secciones del Cuadro 2, el rango (columna derecha) y el número de citas (columna izquierda) en los tres momentos registrados, en orden temporal (agosto de 2012; febrero de 2013; mayo de 2013).

La distribución por universidad o centro de investigación de los artículos más citados está registrada en el Cuadro 3:

Cuadro 3. Distribución de los documentos más citados por institución

\begin{tabular}{|l|c||c|}
\hline INSTITUCIÓN & Frecuencia & Porcentaie \\
\hline \hline Universidad Autónoma de Puebla & 52 & 51.0 \\
INAOE & 39 & 38.2 \\
Universidad Autónoma de Puebla / INAOE & 4 & 3.9 \\
Universidad de las Américas - Puebla & 4 & 3.9 \\
Hospital para el Niño Poblano & 1 & 1.0 \\
Centro de Hematología y Medicina Interna de Puebla & 1 & 1.0 \\
Investigación aplicada, S.A. de C.V., Tehuacán & 1 & 1.0 \\
Total & 102 & 100.0 \\
\hline
\end{tabular}

Las instituciones aparecen en la columna izquierda en orden descendente, según la frecuencia de documentos más citados. La colaboración interinstitucional en Puebla es aún poco frecuente, pero en el Cuadro 3 está presente ya en cuatro artículos (UAP/INAOE).

Publicaciones con mayor impacto de la Universidad Autónoma de Puebla

Destaquemos que la Universidad Autónoma de Puebla (UAP) ${ }^{8}$-institución pública de educación e investigación-, el Instituto Nacional de Astrofísica, Óptica y Electrónica (INAOE) -centro de investigación público situado en Tonanzintla, Puebla- son los espacios que albergan a los autores más citados. En cuanto a la primera de estas instituciones, los grupos de investigadores se distribuyen de acuerdo con el Cuadro 4, mientras que los autores de estos textos se concentran en el Cuadro 5:

8 A la Universidad Autónoma de Puebla (UAP) recientemente se le antepuso el reconocimiento de "Benemérita" (BUAP), de tal manera que en los documentos publicados es posible encontrar cualquiera de las dos denominaciones. Empleamos en este documento la primera designación. 
CARACTERISTICAS DE LA PRODUCCIÓN CIENTÍFICA DE INSTITUCIONES DE PUEBLA. . .

Cuadro 4. Distribución de grupos o departamentos de investigación de la UAP por frecuencia de documentos

Grupo / Departamento / Instituto Frecuencia Porcentaje

CMS Collaboration

ALICE Collaboration

Pierre Auger Collaboration

Instituto de Ciencias

Instituto de Física

Instituto de Fisiología

Centro de Investigaciones en Ciencias Microbiológicas

Departamento de Farmacia

Facultad de Ciencias Físico Matemáticas

Facultad de Medicina

Total

\begin{tabular}{|c|c|}
21 & 37.5 \\
13 & 23.2 \\
11 & 19.6 \\
3 & 5.4 \\
2 & 3.6 \\
2 & 3.6 \\
1 & 1.8 \\
1 & 1.8 \\
1 & 1.8 \\
1 & 1.8 \\
56 & 100.0 \\
\hline
\end{tabular}

De las 56 comunicaciones en las que participan investigadores adscritos a la UAP, mostradas en los Cuadros 3, 4 y 5, dos son resultado de investigaciones realizadas sin colaboración con otras instituciones (Martínez et al., 2009; Rodríguez et al., 2008), una en colaboración con instituciones de México (Liu et al., 2008) y 53 con participación internacional. En el Cuadro 5 se registra por nombre a los autores de la UAP, con el correspondiente número de textos que suscriben.

Cuadro 5. Distribución del número de artículos por profesor-investigador de la UAP

\begin{tabular}{|c|c|}
\hline Autores de la Universidad Autónoma de Puebla & Frecuencia \\
\hline H.A. Salazar Ibarguen & 21 \\
\hline $\begin{array}{l}\text { I. Cortés Maldonado, A. Fernández Téllez, H. González Santos, M.I. Martínez, J. Muncz, M. Rodriguez } \\
\text { Cahuantzi, G. Tejeda Mưfioz, A. Vargas, S. Vergara }\end{array}$ & 5 \\
\hline $\begin{array}{l}\text { I. Cortés Maldonado, A. Fernandez Téllez, H. Gonzalez Santos, R. López Ramírez, M.I. Martínez, J. } \\
\text { Munoz, M. Rodriguez Cahuantzi, S. Román Lopez, G. Tejeda Muñoz, A. Vargas, S. Vergara }\end{array}$ & 4 \\
\hline R. López O. Martínez Bravo, C. Robledo H. Salazar, I. Torres & 4 \\
\hline R. López, Martinez Bravo, E. Moreno, C. Robledo. H. Salazar & 3 \\
\hline R. López, O. Martínez Bravo, C. Robledo, H. Salazar, I. Torres, A. Zepeda & 2 \\
\hline $\begin{array}{l}\text { A. Fémandez Téllez, R. López-Ramírez, M.I. Martínez, J. Munoz, M. Rodriguez Cahuantzi, S. Román- } \\
\text { López, G. Tejeda Muncz, M.A. Vargas, S. Vergara }\end{array}$ & 1 \\
\hline C. Lara & 1 \\
\hline F. Avelino & 1 \\
\hline F.M Izrailev, A.A. Krokhin & 1 \\
\hline G. Alquicer, G. Flores & 1 \\
\hline G. Rodriguez Zurita, C. Meneses Fabian, N.I. Toto Arellano, J.F. Vázquez Castillo, C. Robledo Sánchez & 1 \\
\hline H. Salazar & 1 \\
\hline $\begin{array}{l}\text { I. Cortés Maldonado, A. Fernandez Téllez, R. López Ramirez, M.I. Martinez hemández, M. Rodriguez } \\
\text { Cahuantzi, S. Román Lopez, G. Tejeda Mufioz, A. Vargas, S. Vergara }\end{array}$ & 1 \\
\hline J. Castro, A. Cordero, J. Cuautle, R. López, Martínez Bravo, C. Robledo, H. Salazar, I. Torres & 1 \\
\hline M. Islas Islas & 1 \\
\hline
\end{tabular}




\begin{tabular}{|l|c|}
\hline Autores de la Universidad Autónoma de Puebla & Frecuencia \\
\hline \hline O. Martínez Bravo. C. Robledo, H. Salazar, I. Torres & 1 \\
\hline R. Martinez Tellez, E. Hemandez Torres, C. Gamboa, G. Flores & 1 \\
\hline T. Scior. J. A. Yunes Rojas & 1 \\
\hline U. Pal & 1 \\
\hline V. Canoa Román, I. Cortés Maldonado, A. Femández Téllez, M.I. Martínez Hemández, M. Rodriguez \\
Cahuantzi, G. Tejeda Muñoz A. Vargas, S. Vergara & 1 \\
\hline V.N. Serkin & 1 \\
\hline V.N. Serkin. T.L. Belvaeva & 1 \\
\hline Total & 56 \\
\hline
\end{tabular}

El Cuadro 5 enlista, en orden descendente, por producción académica, a los autores adscritos a esta institución.

\section{Publicaciones en el ÁREA de FÍsica}

Como se advierte en los Cuadros 4 y 5, un elevado porcentaje (80.36\%) de esas publicaciones se debe a contribuciones en tres relevantes proyectos internacionales: la "Colaboración Pierre Auger" y dos investigaciones del Centro Europeo de Investigaciones Nucleares (CERN, por sus siglas en francés) en dos de sus detectores, el Detector Solenoide Compacto de Muones (CMS, por sus siglas en inglés) y el Experimento del Gran Colisionador de Iones (ALICE, por sus siglas en inglés). En las siguientes líneas comentamos brevemente esos proyectos.

\section{Colaboración Pierre Auger}

Por parte de la UAP participan en este proyecto R. López, O. Martínez Bravo, E. Moreno, C. Robledo, H. Salazar, I. Torres y A. Zepeda. En cuanto al Observatorio Pierre Auger -localizado en la ciudad de Malargüe, Argentina-, el artículo más citado (Abraham et al., 2008a) (203/224/230 citas) menciona que el observatorio es un detector híbrido de superficie que consta de 1,600 detectores Cherenkov separados por una distancia de $1.5 \mathrm{~km}$ y 24 telescopios de fluorescencia, que cubren una superficie de $3,000 \mathrm{~km}^{2}$. Este observatorio emplea dos técnicas independientes para detectar y estudiar rayos cósmicos de alta energía. Uno de ellos detecta partículas de alta energía, a través de su interacción con el agua colocada en tanques. La otra técnica sigue el desarrollo de las cascadas atmosféricas, mediante la observación de la luz ultravioleta emitida en la atmósfera de la Tierra.

Los autores hacen hincapié en que uno de los resultados más destacados obtenido de los datos recolectados por el proyecto del observatorio Pierre Auger proporciona evidencia de la anisotropía en las direcciones de arribo de los rayos cósmicos de alta energía, que se correlacionan con las posiciones 
de los núcleos galácticos activos (AGN) cercanos. Esta correlación es compatible con la hipótesis de que los rayos cósmicos de alta energía provienen de fuentes extragalácticas, que se encuentran lo suficientemente cerca de nuestra galaxia para que su flujo no sea atenuado por la interacción con la radiación cósmica de fondo.

\section{Proyectos ALICE y CMS}

Las grandes colaboraciones que se concentran en el CERN (CMS, ALICE, ATLAS) cuentan con más de tres mil miembros cada cual, procedentes de más de 180 instituciones de alrededor de cuarenta países (Bosman y Anoro, 2012). Estas colaboraciones han permitido analizar una gran cantidad de datos en relativamente poco tiempo y desarrollado poderosos medios gracias al sistema de computación distribuida grid del LHC (Large Hadron Collider o Gran Colisionador de Hadrones) en el que participan todas esas instituciones.

El proyecto CMS es un detector diseñado para observar una amplia gama de partículas y fenómenos producidos en colisiones de alta energía en el LHC. Fue diseñado principalmente para estudiar colisiones protón-protón (y de plomo-plomo). Al lector le interesará saber que el detector CMS tiene una longitud aproximada de $21.6 \mathrm{~m}$, un diámetro de $14.6 \mathrm{~m}$ y un peso total de 12,500 t. Sus principales características son un solenoide, una estructura de rastreo interno de sílice semiconductor y un calorímetro electromagnético de cristales centelladores.

El sistema de coordenadas del CMS tiene su origen centrado en el punto de colisión dentro del experimento, el eje $y$ apuntando verticalmente hacia arriba y el eje $x$ apuntando radialmente hacia el centro del LHC. Así, los puntos a lo largo del eje z están en la dirección del haz. Cuando ocurre una colisión, las partículas viajan a través del CMS dejando patrones característicos o firmas en las diferentes capas, lo que permite identificarlas y de esa manera inferir la presencia de partículas desconocidas hasta ahora y, sin embargo, ya postuladas teóricamente. Todas estas investigaciones se nutren particularmente de la visión de Higgs, Englert y Brout, quienes evaluaron la existencia de una partícula que podría explicar el surgimiento de la masa, propuesta cuya relevancia fue reconocida con el Premio Nobel en Física 2013 para los dos primeros autores.

Un ejemplo, de los más citados entre los 21 artículos indicados en el Cuadro 4, tiene como temática la búsqueda de supersimetría en el LHC (CMS collaboration, 2011i): el experimento CMS realizó, entre el conjunto de datos recolectado de las colisiones protón-protón a una energía del centro de masa de 7 teraelectronvoltios $(\mathrm{TeV})$, una búsqueda de eventos con jets y con energía 
transversal perdida, que corresponde a una luminosidad integrada de $1.14 \mathrm{fb}^{-1}$ (98/114/115 citas). En estos datos fueron analizados los estados finales con dos o más dijets y la energía tranversal significativa como se esperaba para la producción y decaimiento del gluino, pareja fermiónica supersimétrica del gluón, y las masas del squark, contraparte bosónica supersimétrica de los quarks. Los límites sobre los parámetros del modelo estándar mínimo supersimétrico constreñido (CMSSM) fueron derivados, lo cual implica una fuerte restricción sobre el espacio de parámetros en relación con los modelos de Súper Simetría (Susy) y CMSSM.

Por otro lado, destaca el documento sobre el bosón de Higgs del modelo estándar (CMS collaboration, 2012a) que publicó CMS el 29 de marzo de 2012, ya que alcanzó en febrero de 2013217 citas, y en mayo 244; mientras que en agosto de 2012 contaba sólo con 28 citas registradas, lo cual le llevó desde la posición 74 a la 5 (Cuadro 2). Dicho trabajo analiza los resultados combinados de colisiones protón-protón, para cinco diferentes modos de decaimiento del bosón de Higgs. El rango de exploración fue de 100 a 600 GeV. El mayor exceso de eventos se observó para una masa hipotetizada del bosón de Higgs de $124 \mathrm{GeV}$, lo cual es una base para la determinación de las características y la existencia misma de esta partícula. Entre los profesores y estudiantes de la UAP que han colaborado en el proyecto CMS, cabe mencionar a Salazar Ibargüen, Pedraza Morales y Xoxocotzi Aguilar.

En relación con el experimento ALICE, uno de los artículos más citados (ALICE collaboration, 2008) (165/220/231) describe con detalle los componentes del detector del mismo nombre, que consta de un detector de iones pesados en el LHC del CERN -cuya dimensión es de $\mathrm{m}^{3}$ y tiene un peso aproximado de 10000 t-. Según el artículo, el propósito del proyecto es investigar la cromodinámica cuántica o QCD. ALICE está diseñado tanto para estudiar la física de las interacciones fuertes, como el plasma quark-gluón a densidades y energías muy altas en colisiones núcleo-núcleo. Además de observar colisiones con iones de plomo, también analiza corridas con iones más ligeros a menor energía y estudia colisiones protón-núcleo.

El experimento consta de 18 sistemas de detección diferentes, cada uno con tecnología específica, impulsadas tanto por los requerimientos de la física, como por las condiciones experimentales previstas en el LHC. El diseño es riguroso pues debe hacer frente a la multiplicidad de partículas previstas en colisiones $\mathrm{Pb}-\mathrm{Pb}$. Los distintos subsistemas han sido optimizados para ofrecer una buena resolución, así como una excelente identificación de partículas. Tal como lo señalan los autores, esto permitirá estudiar detalladamente a los hadrones, electrones, muones y fotones producidos en la colisión de núcleos pesados. Por parte de la UAP participan I. Cortés Maldonado, 
A. Fernández Téllez, H. González Santos, R. López Ramírez, M.I. Martínez Hernández, J. Muñoz, M. Rodríguez Cahuantzi, S. Román Lopez, G. Tejeda Muñoz, A. Vargas y S. Vergara.

Con un número importante de citas (96/138/158), otro trabajo de este proyecto (ALICE collaboration, 2011b) reporta las primeras mediciones de los coeficientes de flujo anisotrópicos de partículas cargadas en colisiones $\mathrm{Pb}-\mathrm{Pb}$. Los datos fueron recolectados durante la primera corrida con iones pesados en LHC. Se mostró, entonces, qué flujo triangular sería descrito en términos de la anisotropía espacial inicial y sus fluctuaciones, lo cual provee, según los autores, fuertes restricciones sobre su origen.

\section{Sistemas de potenciales aleatorios unidimensionales}

Félix Izrailev Mikhailovich del Instituto de Física participa en una publicación apoyada por la Deutsche Forschungsgemeinschat (dfg) y escrita en colaboración con Ulrich Kuhl y Arkadii Krokhin (Kuhl et al., 2008). En ese documento se muestra que las correlaciones de alto rango incrementan la localización de Anderson en sistemas de potenciales aleatorios unidimensionales. Para llegar a este resultado, los investigadores utilizan un sistema de guía de onda con cien dispersores cilíndricos que es cerrada por dos mecanismos absorbentes de microondas.

\section{INVESTIGACIONES EN NEUROCIENCIAS}

El Instituto de Fisiología, uno de los de mayor producción en la UAP, desarrolla varias líneas de estudio con impacto en los últimos años. Aquí destacaremos el trabajo del grupo de investigación que encabeza Gonzalo Flores. Un documento (Alquicer et al., 2008) (25/30/33 citas) describe los resultados de un proyecto realizado en colaboración con el Douglas Mental Health University Institute y el Departamento de Psiquiatría de la Universidad McGill, ambos en Montreal, Canadá. En este artículo, los autores concluyen que el aislamiento social posterior al destete de ratas neonatales, que han sido lesionadas en el hipocampo ventral de manera excitotóxica bilateral, produce un incremento en los cambios morfológicos. Este modelo de lesión neonatal es adecuado para el estudio de alteraciones conductuales, que aparecen después de la pubertad en las ratas, análogas a las de la esquizofrenia en humanos. En estos animales aislados, la disminución en la longitud de las dendritas de la corteza prefrontal y el núcleo accumbens (NA) se exacerba, al tiempo que se observa un incremento en la densidad de espinas dendríticas en neuronas del NA. 
Como fruto del trabajo de este laboratorio encontramos, entre los textos más citados aquí revisados (30/38/43 citas), un reporte de investigación (Martínez et al., 2009) sobre los efectos del estrés prenatal producido en las ratas madres por restricción de su movilidad durante dos horas diarias a partir del día once de la gestación. Comparados los hijos machos con ratas control, que no fueron sometidas a ninguna forma externa productora de estrés, se observó lo siguiente: el estrés prenatal en ratas afecta la densidad de espinas dendríticas y las dendritas basales de las neuronas piramidales del hipocampo, así como la morfología de las dendritas de las neuronas del NA. Esto puede producir cambios importantes en la transmisión dopaminérgica mesocorticolímbica y conductas vinculadas con el desarrollo de alteraciones como la esquizofrenia en humanos.

\section{Proyectos De Óptica}

\section{Interferometría de corrimiento de fase}

G. Rodríguez Zurita, C. Meneses Fabián, N.I. Toto Arellano, C. Robledo-Sánchez y J.F. Vázquez-Castillo, la mayoría miembros del cuerpo académico de Óptica de la Facultad de Ciencias Físico Matemáticas son los responsables de uno de los trabajos más citados, realizados sin colaboración externa a su departamento (Rodríguez et al., 2008). Asimismo, son responsables de una publicación electrónica de nombre Tlabuizcalpan, cuyo objetivo es la divulgación de la ciencia y de la investigación aplicada. Ellos han desarrollado un sistema de interferometría de corrimiento de fase con rejilla de fase en un solo disparo o toma. El sistema, que emplea una rejilla de difracción 2D, puede adquirir múltiples interferogramas.

En contraste con el procedimiento secuencial de la metodología llamada Corrimiento de Fase, el sistema propuesto por Rodríguez Zurita y colaboradores es una técnica para evaluar frentes de onda ópticos de manera no secuencial. En palabras de los autores:

para implementar la evaluación no secuencial, se echa mano de una rejilla de difracción, pero no de absorción, sino de fase. Una rejilla como la empleada (rejilla de fase) es el componente óptico denominado Phase Grating que consiste en una serie de líneas paralelas en relieve y transparentes. Las líneas tienen separación constante y producen difracción de modo conveniente. Cuando se forma un tramado o malla, cruzando por ejemplo dos rejillas con sus líneas a 90 grados, se obtiene una malla de fase, que es la llamada Phase Grid 
(comunicación vía correo electrónico con el Doctor Rodríguez Zurita, 8 de junio de 2013).

Este método reduce los errores de medición ocasionados por factores medioambientales, aunque resulta más apropiado con objetos pequeños que no introduzcan cambios de polarización.

\section{Solitones}

Vladimir N. Serkin ha realizado investigaciones en colaboraciones internacionales acerca de la dispersión y propagación de solitones. Serkin ha propuesto nuevos métodos de generación de pulsos láser ultracortos y solitones ópticos. El concepto de solitón permite caracterizar ondas solitarias no lineales, que no se dispersan y preservan su identidad durante su propagación después de alguna colisión. Por sus características, ha sido considerado el modelo ideal de bits naturales de datos. El solitón es la única forma estable en una fibra con dispersión y no linealidad para un amplio rango de amplitud de pulsos y un pico de poder.

En un trabajo realizado en colaboración con investigadores de India y Japón (Ganapathy et al., 2008) (34/35/35 citas), Serkin analiza algunas características de las interacciones de solitones de igual amplitud y de aquellas con pulsos de solitones de amplitudes desiguales, cuyas velocidades son iguales en magnitud, pero opuestas en signo. En ese estudio se subraya el papel del control de la dispersión, en el cual el solitón puede ser controlado y estructurado por sus cuatro parámetros básicos: amplitud, frecuencia, fase y posición temporal. En el documento se examinan las fuerzas de interacción de solitones, la amplificación de los pulsos después de la colisión, el logro de la amplificación óptima, así como los efectos de la interacción de ondas no lineares de Boch. El segundo documento (Serkin et al., 2010) (30/35/39 citas), escrito por Serkhin y Belyaeva de la UAP, en colaboración con Hasegawa de Japón, considera los rasgos principales de solitones no autónomos materia-onda cercanos a la resonancia Feshbach, en un condensado unidimensional Bose-Einstein confinado por un potencial harmónico.

\section{INVESTIGACIONES EN CIENCIAS QUÍMICAS}

\section{Fusión de nanogrupos oro-platino}

Umapada Pal, en colaboración con investigadores del Instituto Mexicano del Petróleo y de la unam, publicó en 2008 un trabajo (Liu et al., 2008) (24/30/30 
citas) sobre la estabilidad termodinámica y la fusión de nanogrupos Au-Pt con las variantes estructurales más comunes. Los grupos bimetálicos adquieren la estructura más estable con centro de oro y coraza de platino, independientemente de la estructura inicial por encima de ciertas temperaturas. Consideran, asimismo, que la gran diferencia entre los puntos de fusión de los elementos constituyentes sería un factor determinante sobre los mecanismos de fusión de las nanopartículas bimetálicas. Pal ha desarrollado, entre otras, una línea de investigación sobre nanocompositos o nanocompósitos.

\section{INVESTIGACIÓN EN PSIQUIATRÍA}

\section{Persistencia del sindrome de déficit de atención con hiperactividad}

El trabajo en el que participa Carmen Lara, por parte de la UAP, es un proyecto de colaboración internacional y forma parte de la iniciativa de análisis sobre Salud Mental Mundial (smm) de la Organización Mundial de la Salud (OMS). El documento (Lara et al., 2009), escrito por dieciséis coautores (con 39/48/52 citas), evalúa la persistencia de la niñez a la edad adulta del síndrome de déficit de atención con hiperactividad (ADHD) y los factores que pueden predecir esa continuidad en muestras multinacionales.

\section{PubliCACIONES EN CIENCIAS BIOMÉdiCAS}

\section{Formación de biopelículas y la adherencia bacterial}

Fabiola Avelino, con adscripción al Instituto de Ciencias, en colaboración con investigadores del Reino Unido, Estados Unidos y del Instituto de Biotecnología de la unam, en una comunicación científica (Saldaña et al., 2009) (33/39/46 citas) publicada en 2009, concluye que la producción de fibras de curli y celulosa interaccionan sincronizadamente, favoreciendo la formación de biopelículas e incrementando la adherencia bacterial. Estos resultados fueron observados en E. coli enterohemorrágica (EHEC) y enteropatógénica (EPEC). La proteína CsgA es la subunidad mayor de las fibras de curli y la subunidad catalítica celulosa sintetasa (gen $b s c A$ ) es esencial en la formación de celulosa. La expresión de fibras de curli (gen $\operatorname{csg} A$ ) y de celulosa son co-activados por el regulador transcripcional CsgD. Los autores hicieron comparaciones en las propiedades de adherencia en mutantes sencillas de $\operatorname{csg} A, \operatorname{csg} D$ (gen activador de $\operatorname{csg} A B$ ), y $\operatorname{bsg} A$ (proteasa La dependiente de ATP) de EHEC y EPEC. Asimismo, estudiaron el efecto de la sobreexpresión de $\operatorname{csg} D$ en estos tipos mutantes. Finalmente, sus datos los llevan a concluir que el Fis (factor 
para la estimulación de la inversión) actúa como un regulador transcripcional negativo de $\operatorname{csg} A$.

\section{Evaluación de la capacidad de cepas de E. coli para inducir la inflamación en dos lineas de células intestinales}

Martha Islas Islas del Centro de Investigaciones Microbiológicas del Instituto de Ciencias, en coautoría con siete investigadores de Estados Unidos y Alemania, en una comunicación científica (Eaves et al., 2008) (29/30/34 citas) refieren que la cepa de E. coli O83:H1 posee las características de la nueva categoría denominada E. coli Adherente Invasiva (AIEC). Esta cepa, junto con otras tres, fue aislada de pacientes con enfermedad de Crohn para evaluar su capacidad para inducir la inflamación en dos líneas de células intestinales (Caco-2BBe y T84). Básicamente, la O83:H1 fue la que más se asemeja a la cepa LF82 prototipo de la AIEC.

\section{Limitaciones y requisitos para una adecuada aplicación de QSAR}

Los métodos y modelos de cuantificación de las relaciones entre la estructura molecular y la actividad biológica (QSAR, por sus siglas en inglés) han alcanzado un alto valor en la predicción y diagnóstico en medicina y química. T. Scior y A. Yunes Rojas, en colaboración con investigadores de Estados Unidos y Francia, en un texto de 2009 (26/33/39 citas) analizan las limitaciones y los requisitos para una adecuada aplicación de QSAR (Scior et al., 2009). Varias alternativas han sido propuestas para afrontar las deficiencias comunes en la aplicación de QSAR. Asimismo, son discutidos los aspectos que generarían problemas en estos modelos, como el tamaño y composición de los datos, la validación interna y externa de los modelos, los supuestos de linealidad y una adecuada interpretación de los valores que salen del ajuste (outliers).

\section{Características de autoría de las publicaciones de la UAP}

Derivados, en su mayoría, de colaboraciones en proyectos internacionales como los descritos hasta aquí, llama la atención que los trabajos de la UAP con mayor cantidad de citas sean producto de la coautoría de más de cien personas. Esta característica es puesta de manifiesto en el Cuadro 6. 
Cuadro 6. Relación de número de autores (UAP) y número de citas

\begin{tabular}{|l|c|c|c|c|c|}
\hline \multirow{2}{*}{$\begin{array}{c}\text { Número de } \\
\text { autores UAP }\end{array}$} & \multicolumn{4}{|c|}{ Número de citas } & \multirow{2}{*}{ Total } \\
\cline { 2 - 6 } & $\begin{array}{c}\text { más de 200 } \\
\text { citas }\end{array}$ & $\begin{array}{c}100 \text { a } 199 \\
\text { citas }\end{array}$ & 50 a 99 citas & 30 a 49 citas & \\
\hline más de 1900 & 1 & 2 & 5 & 13 & 21 \\
900 a 1100 & 0 & 1 & 8 & 4 & 13 \\
100 a 599 & 2 & 1 & 5 & 2 & 10 \\
11 a 20 & 0 & 0 & 0 & 2 & 2 \\
3 a 10 & 0 & 0 & 0 & 10 & 10 \\
\hline Total & 3 & 4 & 18 & 31 & 56 \\
\hline
\end{tabular}

\section{Publicaciones CON MAYOR IMPACto del INAOE}

En cuanto al INAOE, la responsabilidad de los documentos más citados recae en un pequeño número de investigadores, ya que, como se indica en el Cuadro 7, los 43 trabajos ahí concentrados se deben a un número reducido de investigadores.

\section{Cuadro 7. Distribución del número de artículos por investigador del INAOE}

\begin{tabular}{|l|c}
\hline Autores INAOE & Documentos \\
\hline \hline D.H. Hughes & 18 \\
\hline I. Aretxaga, D.H. Hughes & 7 \\
\hline A. Bress an & 2 \\
\hline M. Plionis & 2 \\
\hline R. López, Martínez Bravo, E. Moreno, C. Robledo, H. Salazar & 2 \\
\hline R. López, O. Martínez Bravo, C. Robledo, H. Salazar, I. Torres, A. Zepeda & 2 \\
\hline O. Vega, A. Bressan & 1 \\
\hline I. Aretxaga & 1 \\
\hline I. Aretxaga, D.H. Hughes, D. Ferrusca, M. Velazquez & 1 \\
\hline $\begin{array}{l}\text { E. Aguilar, I. Aretxaga, D. Ferrusca, D.H. Hughes, M. Velazquez, M. } \\
\text { Zeballos }\end{array}$ & 1 \\
\hline J. Wagg, I. Aretxaga, D. Hughes & 1 \\
\hline A. Porras & 1 \\
\hline H.F. Hägele, M.V. Cardaci & 1 \\
\hline J.C Ramirez San Juana, R. Ramos Garcia, I. Guizar Iturbide, G. Martinez \\
Niconoff & 1 \\
\hline S. Chávez-Cerda & 1 \\
\hline Y. Juarez, R. Mujica & 1 \\
\hline Total & 43 \\
\hline
\end{tabular}




\section{Investigaciones en astronomía, astrofísica y óptica}

Iniciaremos la revisión de este instituto con los trabajos publicados por $\mathrm{Hu}$ ghes, Aretxaga, Porras y Bressan. Los dos primeros son coautores de más de la mitad de estos artículos, y el último es coautor del documento con el mayor número de citas (Marigo et al., 2008) (443/524/549 citas).

Este artículo provee el primer conjunto de isócronas teóricas cuyos rasgos distintivos residen fundamentalmente en un tratamiento perfeccionado del estudio de las estrellas en la fase térmicamente pulsante de la rama asintótica gigante (tp-agb, por sus siglas en inglés). Las isócronas teóricas tienen un amplio rango de aplicaciones que van desde el cálculo de la edad de poblaciones estelares, hasta la solución de problemas más complejos (Marigo et al., 2008). Por ejemplo, se utilizan para calcular la evolución espectral de poblaciones estelares. Esto permite el desarrollo de modelos teóricos de formación y evolución estelar. Una isócrona especifica la ubicación de estrellas con la misma edad y metalicidad en el diagrama Hertzsprung-Russell.

David $H$. Hughes, director del proyecto e investigador principal del Gran Telescopio Milimétrico, es corresponsable de veintiocho de los artículos del INAOE aquí revisados. Destaca su investigación en la astronomía e instrumentación milimétrica, en especial acerca de la formación y evolución de las grandes estructuras del universo: cúmulos de galaxias y galaxias en formación.

Itziar Aretxaga es coautora de once de los artículos con más citas. Sus áreas de investigación dentro de la astrofísica y la cosmología son el estudio de las galaxias en formación, las supernovas, así como la simbiosis entre la formación estelar y la actividad nuclear de galaxias.

Los proyectos de investigación en que participan Hughes, Aretxaga, Aguilar, Ferrusca, Velázquez, Wagg y Zeballos tienen como objetivo central determinar la composición de las poblaciones de astros que producen la mayor parte de la emisión energética del universo, lo cual permitirá esclarecer la historia de formación y evolución de las poblaciones de galaxias que dominan la emisión del fondo extragaláctico.

Por ejemplo, al parecer, la emisión en luz desde la infrarroja lejana a la milimétrica del universo es producida, en un buen porcentaje, por la formación estelar violenta de galaxias masivas en formación fuertemente oscurecidas. Gracias al consorcio SHADES (por las siglas en inglés de Submillimetre Common-User Bolometer Array (SCUBA) Half Degree Extragalactic Survey) ha sido posible realizar una cartografía milimétrica más extensa -por ejemplo, con más de cincuenta citas hallamos dos trabajos: uno 
de ellos (Coppin et al., 2008) con 68/72/72 citas y otro (Dye et al., 2008) con 53/55/55 citas-. Este consorcio cuenta con la participación de trece institutos de Reino Unido, México, Estados Unidos, Canadá, Italia, España, Austria y Japón.

El desarrollo de instrumentos que permiten manipular la luz y la registran en formato digital potencian las capacidades de los telescopios. La fotometría adquirida con las cámaras SCUBA a $0.85 \mathrm{~mm}$ y AZTEC a $1.1 \mathrm{~mm}$ revelan una población de galaxias hasta ahora no detectables. AZTEC, una cámara bolométrica, fue desarrollada en la Universidad de Massachusetts, en colaboración con el Instituto de Tecnología de California, la Universidad de Cardiff, el INAOE, la Universidad Sejong y el Smith College. Esta cámara, usada tanto en los telescopios ASTE (Atacama Submillimeter Telescope Experiment), como en el JCMT (James Clerk Maxwell Telescope) ha permitido un análisis de densidad y distribución espacial de la población de fuentes milimétricas hacia diecisiete galaxias activas a altos corrimientos al rojo usando observaciones a $1.1 \mathrm{~mm}$. Encontramos dos reportes (Scott et al., 2008; Perera et al., 2008), derivados de estas investigaciones entre los más citados del periodo aquí estudiado y registrados en el Cuadro 2.

Los experimentos y observaciones del telescopio submilimétrico Atacama son, igualmente, resultado de proyectos internacionales en los que participa el INAOE. Este telescopio fue instalado a una altura de 5,190 m en Cerro Toco, en el desierto de Atacama, al norte de Chile, en 2007. El tercer documento más citado en el que colabora algún investigador del INAOE (Hughes) tiene que ver con los parámetros cosmológicos establecidos sobre la base de datos obtenidos en Atacama (Dunkley et al., 2011) (65/122/145 citas).

Asimismo, incluimos en el Cuadro 2 escritos que versan sobre los datos obtenidos en el proyecto Herschel-ATLAS con el telescopio del observatorio espacial Herschel (Amblard et al., 2010; Eales et al., 2010). Vinculados con estos datos se encuentran los hallazgos realizados con BLAST, un telescopio estratosférico de $1.8 \mathrm{~m}$ que opera a una altitud aproximada de $35 \mathrm{~km}$. BLAST es un precursor del SPIRE (Spectral and Photometric Imaging Receiver) del observatorio espacial Herschel. En cuanto a los resultados obtenidos, se publicó un artículo en la revista Nature (Devlin et al., 2009) (99/105/105 citas). Otro documento (Marsden et al., 2009) relacionado con las investigaciones desarrolladas con BLAST y que recibió un número importante de citas (62/70/71) fue publicado en la revista Astrophysical Journal.

Con un número importante de citas $(117 / 132 / 135)$ se encuentra el artículo suscrito, entre otros, por Alicia Porras, investigadora del INAOE (Gutermuth et al., 2008). Este texto ofrece un conjunto de datos obtenido por el telescopio espacial Spitzer, el cual es una misión de la NASA operada y 
administrada por el Laboratorio de Propulsión a Reacción, mantenido por el Grupo de Educación y Difusión Pública en el Centro Científico Spitzer, localizado en el Instituto de Tecnología de California, y que forma parte del Centro de Análisis y Procesamiento Infrarrojo (IPAC) de la NASA. En el documento citado se analizan los datos obtenidos de la NGC 1333 con los instrumentos de Spitzer: IRAC (por sus siglas en inglés: Infrared Array Camera) y MIPS (por sus siglas en inglés: Multiband Imaging Photometer).

Entre los trabajos del INAOE, cabe mencionar uno que versa sobre el desarrollo de tecnología biomédica para registrar y fiscalizar el flujo sanguíneo y su dinámica de perfusión (Ramírez et al., 2008); un segundo que trata sobre el análisis de los patrones de difracción de haces luminosos, dependientes del momento angular orbital del haz incidente en una apertura triangular y cómo este efecto puede ser utilizado para medir la carga topológica del haz (Hickmann et al., 2010).

Finalmente, dos artículos en los que Manolis Plionis, coautor por el INAOE, analiza de manera sistemática trece diferentes procedimientos de localización de vacíos (voids; amplias regiones del espacio escasamente pobladas por galaxias) (Colberg et al., 2008), y otro texto que analiza las propiedades de modelos cosmológicos planos Friedmann-Lemaître-Robertson-Walker (FLRW) (Basilakos et al., 2009).

\section{Características de autoría de las publicaciones del INAOE}

Respecto del INAOE, el Cuadro 8 muestra la relación de coautorías y número de citas de los artículos en los que participan investigadores pertenecientes a esta institución.

Cuadro 8. Relación de número de autores (INAOE) y número de citas

\begin{tabular}{|l|c|c|c|c|c|}
\hline \multirow{2}{*}{$\begin{array}{c}\text { Número de autores } \\
\text { INAOE }\end{array}$} & $\begin{array}{c}\text { Nás de 200 } \\
\text { citas }\end{array}$ & $\begin{array}{c}100 \text { a } 199 \\
\text { citas }\end{array}$ & 50 a 99 citas & 30 a 49 citas & Total \\
\cline { 2 - 6 } & 0 & 1 & 2 & 1 & 4 \\
50 a 599 & 0 & 1 & 5 & 3 & 9 \\
31 a 49 & 0 & 0 & 1 & 3 & 4 \\
21 a 30 & 0 & 1 & 6 & 5 & 12 \\
11 a 20 & 0 & 0 & 2 & 3 & 5 \\
3 a 10 & 1 & 1 & 0 & 7 & 9 \\
\hline Total & 1 & 4 & 16 & 22 & 43 \\
\hline
\end{tabular}




\section{Publicaciones con mayor impacto de otras instituciones poblanas}

En el Cuadro 9 se encuentran los autores adscritos a otras instituciones, que participan en alguno de los 102 textos del Cuadro 2.

\section{Publicaciones CON MAYOR IMPACTO DE LA UdLAP}

Víctor Vysloukh es profesor del Departamento de Física y Matemáticas de la Universidad de las Américas-Puebla (UDLA-P), institución privada de educación superior. Realiza investigación en proyectos de colaboración con el INAOE, con el Instituto de Ciencias Fotónicas y la Universidad Politécnica de Cataluña, España, así como con el Instituto de Física Aplicada de Jena, Alemania. Es coautor de tres textos que registran un significativo número de citas. En uno de ellos (Szameit et al., 2009) (32/33/35 citas), los autores reportan que los fenómenos resonantes accesibles en estructuras moduladas longitudinalmente abren nuevas maneras para el control de la propagación de la luz. Mientras que en los otros dos textos $-72 / 83 / 85$ citas (Kartashov et al., 2009b) y 28/31/33 (Kartashov et al., 2009a) citas, respectivamente- analizan las características y propiedades de solitones en retículos ópticos.

Cuadro 9. Distribución del número de documentos publicados por autores de instituciones diferentes de la UAP o el INAOE

\begin{tabular}{|l|l||c|}
\hline Autores & Institución & Documentos \\
\hline \hline V.A. Vysloukh & Universidad de las Américas - Puebla & 3 \\
A. Flores Nunez & Hospital para el Niño Poblano & 1 \\
C.A. Martinez-Huitte, A. De Battisti, S. Ferro, & Universidad de las Américas - Puebla & 1 \\
E.L. Decanini & Investigación aplicada, S.A. de C.V., Tehuacán & 1 \\
G.J. Ruiz. Argüelles & Centro de Hematología y Medicina Intema de Puebba & 1 \\
\hline Total & & 7 \\
\hline
\end{tabular}

\section{Publicaciones CON MAYOR IMPACTO DEL CHMI}

Fundado en 1983, el Centro de Hematología y Medicina Interna (CHMI) de Puebla es una institución médica privada orientada al estudio, diagnóstico y tratamiento de enfermedades hematológicas y de medicina interna. De los cuarenta y dos documentos recuperados en nuestra búsqueda, correspondiente al periodo 2008-2012, en cuya autoría se incluye a investigadores de este centro, se encuentra el texto sobre la eficacia en el tratamiento con Alemtuzumab de pacientes con enfermedad del injerto contra el huésped córticorresistente, posterior al trasplante de células troncales hematopoyéticas 
(Gómez et al., 2008) (28/33/34 citas). Alemtuzumab es un anticuerpo humanizado anti-CD52 dirigido contra el antígeno de superficie CD52, el cual se expresa en linfocitos y monocitos. Este anticuerpo se obtiene mediante técnicas de ADN recombinante.

\section{Publicaciones CON MAYOR IMPACTO DEL HNP}

El Hospital para el Niño Poblano (HNP) es una institución de asistencia a la salud y dedicado a la formación de recursos humanos fomentando la investigación. Proporciona servicios de salud de alta especialidad a la población sin seguridad social menor de dieciocho años. Esta institución aparece en once publicaciones del total de los 3,585 textos ya mencionados. De ellos destaca el que se refiere a la evaluación de la eficacia de montelukast (Bisgaard et al., 2008), un bloqueador del receptor de leucotrieno en el tratamiento de síntomas respiratorios recurrentes que se presentan en niños que padecen bronquiolitis, debida al virus sincitial respiratorio (30/36/36 citas).

Publicaciones con mayor impacto de Investigación Aplicada, S.A. de C.V.

Eduardo Lucio Decanini ha sido director industrial de Investigación Aplicada, S.A. de C.V., Tehuacán, Puebla, empresa mexicana de capital privado del ramo farmacéutico veterinario, que forma parte del Grupo IDISA. Dicho centro se ubica en el área de la biotecnología aplicable a la identificación, prevención y solución de problemas en nutrición y salud animal. Decanini, en colaboración con investigadores que trabajan en Estados Unidos, publicó los resultados de un estudio sobre la evolución de los genotipos del virus de la enfermedad de Newcastle y las consecuencias que su diversidad ocasiona en el diagnóstico de esa patología (Miller et al., 2010) (25/38/42 citas).

Cabe destacar el trabajo de otras instituciones del estado de Puebla, como las de sanidad, que realizan investigación de alta calidad. Por ejemplo, en el periodo aquí analizado, investigadores adscritos al Instituto Mexicano del Seguro Social (IMSS) y al Instituto de Seguridad y Servicios Sociales de los Trabajadores al Servicio del Estado de Puebla (ISSSTEP) publicaron un documento con un número importante de citas (24/25/26) (García et al., 2009), en colaboración con investigadores de la UAP, así como de instituciones de Barcelona, Cuenca (Ecuador) y Filadelfia. El artículo es una revisión sobre el uso de rituximab (anticuerpo monoclonal anti-CD20) en pacientes que padecen lupus eritematoso sistémico. 


\section{Indices h y promedios de citas por área de investigación}

Para analizar algunos índices bibliométricos de las publicaciones por áreas temáticas, adoptamos la clasificación general que la WoS asigna a cada documento. De esta manera, las publicaciones consideradas en este estudio se agrupan en las siguientes áreas: Agricultura; Alimentos; Antropología y Arqueología; Artes, Literatura, Filosofía y Humanidades; Astronomía y Astrofísica; Ciencia y tecnología; Ciencias Ambientales; Ciencias Biológicas; Ciencias Biomédicas; Ciencias de la Computación; Ciencias de materiales; Ciencias Sociales; Cristalografía; Derecho y Administración Pública; Educación; Energía; Física; Geología y Geografía; Ingeniería; Instrumentación; Investigación de Operaciones; Matemáticas; Negocios y Economía; Neurociencias; Óptica; Psicología y ciencias de la conducta; Química (ciencias biológicas); Química (materiales); Sistemas de Control y Automatización; Telecomunicaciones.

En el Cuadro 10 se registra, para cada área de investigación, en primer lugar, el índice $h$, enseguida entre paréntesis el promedio de citas de los $b$ documentos que tienen al menos un número $b$ de citas y, finalmente, se indica el número de citas promedio de todos los documentos del área. El índice $b$ hace referencia al número $b$ de publicaciones que han sido citadas por lo menos un número $b$ de veces (Hirsch, 2005). Estos indicadores por sí solos no son indicios totalmente objetivos de la calidad y del impacto de las publicaciones por área, pero pueden, en conjunto, proporcionar una imagen de aquéllos. Por ejemplo, aunque los documentos del área de instrumentación tienen un índice $b$ de siete y su promedio total de citas es de 6.59, de un total de 83 publicaciones, el promedio de citas de los textos que cuentan con al menos siete citas es de 62.57. Este valor supera los promedios de los documentos más citados de las áreas de óptica y ciencias biomédicas (29.75 y 27.94 , respectivamente) que tienen índices $h$ de 16 y 18 , correspondientemente. Consideramos importante señalar que el alto promedio del área de instrumentación es resultado de un artículo de investigación que tiene 231 citas.

Cuadro 10. Promedio de citas por área de investigación

\begin{tabular}{|c|c|c|c|c|c|c|c|c|c|}
\hline $\begin{array}{c}\text { Artes, } \\
\text { Literatura, } \\
\text { Filosoffay } \\
\text { Munumidudes }\end{array}$ & $\begin{array}{l}\text { Cencias } \\
\text { Seciales }\end{array}$ & $\begin{array}{c}\text { Derechioy } \\
\text { Administración } \\
\text { Páblica }\end{array}$ & Cristalografía & \begin{tabular}{|} 
Antropdogiay \\
Arcpedogiagia
\end{tabular} & $\begin{array}{l}\text { Negocios y } \\
\text { Frononiala }\end{array}$ & $\begin{array}{c}\text { Psicologiay } \\
\text { eiencias de la } \\
\text { conducta }\end{array}$ & $\begin{array}{l}\text { Gedogiay } \\
\text { Geograffia }\end{array}$ & $\begin{array}{l}\text { Inustigación de } \\
\text { Operiacienes }\end{array}$ & $\begin{array}{l}\text { Teleconaumicar } \\
\text { ciones }\end{array}$ \\
\hline $2(2) .14$ & $2(4) 1$ & $2(4.5) .82$ & $3(3) .66$ & $3(4) 1.13$ & $3(4.28) .80$ & $3(4,4) \cdot 71$ & $3(5.33) 2.38$ & $3(5.57) 1.69$ & $3(5.75) 2.45$ \\
\hline Educación & $\begin{array}{c}\text { Sistemas de } \\
\text { Controly } \\
\text { Autematizaxion }\end{array}$ & Ehergia & $\begin{array}{c}\text { Clencias } \\
\text { Biologeicas }\end{array}$ & Matemáticas & Agricultura & $\begin{array}{l}\text { Clenelise de la } \\
\text { Compertación }\end{array}$ & Alimentos & Instrume ntación & $\begin{array}{l}\text { Quimica: } \\
\text { materiales }\end{array}$ \\
\hline 30.78 & $3(7), 32$ & $4(8.25) 2.80$ & $5(7.2) 1.15$ & $5(8) 1.22$ & $5(88) 1.22$ & $6(10.37), .77$ & $7(12.14) 3.04$ & $7(62.57) 6.59$ & $8(13.62) 3.15$ \\
\hline $\begin{array}{l}\text { Cienciay } \\
\text { tecudogia }\end{array}$ & $\begin{array}{c}\text { Ciencias } \\
\text { Ambientales }\end{array}$ & Ingenieria & $\begin{array}{c}\text { Ciencias de } \\
\text { materiales }\end{array}$ & Neurocieacias & $\begin{array}{c}\text { Quimicas } \\
\text { Ciencias } \\
\text { Bidolicicas } \\
\end{array}$ & Opxica & $\begin{array}{c}\text { Ciencias } \\
\text { Biomédcas }\end{array}$ & $\begin{array}{c}\text { Astronomiay } \\
\text { Astrofisica }\end{array}$ & Fisica \\
\hline $8(31) 4.22$ & $9(14.5) 2.72$ & $9(15.9) 1.44$ & $10(16.72) 2.65$ & $10(24) 5.10$ & $11(14.57) 3.13$ & $16(29.75) 2.44$ & $18(27.94) 3.17$ & $34(79.8) 12.06$ & $35(91.94) 10.71$ \\
\hline
\end{tabular}


Para cada área de investigación, se registra el índice $h$, a continuación y entre paréntesis, el promedio de citas de los $b$ documentos que tienen al menos un número $b$ de citas y, finalmente, se indica el número de citas promedio de todos los documentos del área.

\section{IMPACTO DE LAS PUBLICACIONES DE UN SOLO AUTOR}

En el Cuadro 11 está indicado el número de citas de los escritos con un solo autor, con seis citas el máximo alcanzado (entre paréntesis se anota el número de autocitas). Del resto de los 33 documentos con un autor que cuentan por lo menos con una cita, siete tienen dos citas que son autocitas y dieciséis registran una autocita.

Cuadro 11. Enumeración de artículos escritos por un autor con tres citas o más

\begin{tabular}{|l|l||c|l|c|c|c}
\hline INSTITUCIÓN & Autor & Año & Revista & Vol. & pag. & Citas* \\
\hline \hline UAP & Castañeda, L. & 2009 & Acta Materialia & 57 & 1385 & $6 / 7(0)$ \\
\hline UAP & Escalante, Alberto & 2009 & Physics Letters B & 676 & 105 & $5 / 7(4 / 6)$ \\
\hline $\begin{array}{l}\text { FAC LAT CCSS \& } \\
\text { UPAEP }\end{array}$ & Rojas, Mariano & 2009 & $\begin{array}{l}\text { Applied Res earch in Quality } \\
\text { of Life }\end{array}$ & 4 & 179 & $5 / 5(2)$ \\
\hline INAOE & Jovanovic Dolecek, G. & 2009 & Electronics Letters & 45 & 1270 & $4 / 7(1)$ \\
\hline UAP & Lorenzo Diaz-Cruz, J. & 2008 & Physical Review Letters & 100 & & $4 / 6(0)$ \\
\hline UAP & Binford, Leigh & 2009 & Third World Quarterly & 30 & 503 & $4 / 5(0)$ \\
\hline INAOE & Barberis-Blos tein, P. & 2008 & Physical Review A & 77 & & $4 / 4(1)$ \\
\hline UDLA-P & Ibarra, Carlos & 2008 & Cepal Review & & 83 & $3 / 3(0)$ \\
\hline $\begin{array}{l}\text { CTR HEMATOL \& } \\
\text { MED INT }\end{array}$ & Ruż-Arguelles, Guillermo J. & 2010 & Hematology & 15 & 1 & $3 / 3(2)$ \\
\hline UDLA-P & Ibarra, Carlos A. & 2008 & Investigacion Economica & 67 & 67 & $3 / 3(2)$ \\
\hline
\end{tabular}

El número de citas sigue el mismo formato que hemos empleado antes, el cual consiste en señalar el número de citas registrado en las dos últimas búsquedas (febrero/mayo) separado por una barra, con la adición del número de autocitas entre paréntesis.

\section{CARACTERÍSTICAS DE LAS PUBLICACIONES EN TÉRMINOS DE IDIOMA}

De los 1,718 documentos que registran al menos una cita, solamente 45 fueron escritos en español: veintisiete de ellos con una sola cita, nueve citados dos veces, cinco tres veces, dos cuatro veces, tan solo un documento citado siete veces y otro más con diez citas. Del total de los 3,585 documentos registrados en el periodo analizado, sólo dos fueron escritos en portugués y otro de ellos en francés, estos tres textos no registran ninguna cita. 


\section{REVISTAS CON EL MAYOR NÚMERO DE CITAS PARA DOCUMENTOS CON PARTICIPACIÓN POBLANA}

Las treinta revistas con más citas acumuladas en las que publicaron investigadores poblanos se muestran en el Cuadro 12.

Cuadro 12. Publicaciones con el mayor número de citas en el periodo 2008-2012

\begin{tabular}{|c|c|c|c|}
\hline \multirow{2}{*}{ Revista } & \multicolumn{3}{|c|}{ Citas } \\
\hline & ago-12 & feb-13 & may -13 \\
\hline Physical Review Letters & $1225(48)$ & $1745(65)$ & 1924(65) \\
\hline Astrophy sical Journal & $1137(72)$ & $1454(82)$ & $1557(82)$ \\
\hline Physics Letters B & $653(39)$ & $1438(59)$ & $1844(59)$ \\
\hline M onthly Notices of the Royal Astronomical Society & $961(71)$ & $1148(77)$ & $1177(77)$ \\
\hline Astronomy \& Astrophysics & $785(32)$ & $960(45)$ & $1008(45)$ \\
\hline Journal of High Energy Phy sics & $320(40)$ & $602(69)$ & $688(74)$ \\
\hline Astrop art icle Physics & $412(14)$ & $512(15)$ & $553(15)$ \\
\hline European Physical Journal C & $295(12)$ & $410(20)$ & $461(21)$ \\
\hline Physical Review D & $236(43)$ & $361(55)$ & $391(55)$ \\
\hline Journal of Instrumentation & $197(6)$ & $310(10)$ & $337(10)$ \\
\hline Optics Letters & $204(29)$ & $236(35)$ & $256(35)$ \\
\hline Optics Express & $174(23)$ & $212(32)$ & $231(33)$ \\
\hline Nature & $122(2)$ & $132(2)$ & $132(2)$ \\
\hline Nuclear Instruments \& M ethods in Physics Research Section A & $86(8)$ & $122(8)$ & $131(8)$ \\
\hline Publicat ions of the Astronomical Society of the Pacific & $72(2)$ & $98(3)$ & $106(3)$ \\
\hline Physical Review A & $77(11)$ & $97(12)$ & $107(12)$ \\
\hline Synapse & $69(16)$ & $93(18)$ & $100(18)$ \\
\hline Physical Review B & $75(13)$ & $87(16)$ & $93(16)$ \\
\hline Astronomical Joumal & $67(11)$ & $82(12)$ & $89(12)$ \\
\hline Progress in Optics, Vol 52 & $72(1)$ & $83(1)$ & $85(1)$ \\
\hline Laser Phy sics & $81(13)$ & $83(15)$ & $83(15)$ \\
\hline Astrophy sical Journal Letters & $52(10)$ & $73(11)$ & $78(11)$ \\
\hline Phy sical Review C & $39(1)$ & $71(1)$ & $79(1)$ \\
\hline Journal of Chemical Neuroanatomy & $53(3)$ & $63(3)$ & $71(3)$ \\
\hline Journal of Phy sical Chemistry C & $43(7)$ & $62(7)$ & $65(7)$ \\
\hline App lied Physics Letters & $48(9)$ & $61(10)$ & $65(10)$ \\
\hline Science & $40(1)$ & $57(1)$ & $65(2)$ \\
\hline Bioresource Technology & $41(4)$ & $52(4)$ & $57(4)$ \\
\hline IEEE Journal of Quantum Electronics & $50(4)$ & $51(4)$ & $51(4)$ \\
\hline Autoimmunity Reviews & $41(5)$ & $51(5)$ & $56(5)$ \\
\hline
\end{tabular}

Nota: entre paréntesis se indica el número de documentos registrados.

\section{ReFLEXIONES FinALES}

Como se desprende de este recorrido por la investigación en Puebla, según el registro de citas en la WoS, la Universidad Autónoma de Puebla y el Ins- 
estado con mayor participación en los documentos más citados del periodo estudiado.

Por lo demás, la producción científica con participación de instituciones poblanas se concentra en las ciencias físicas y biomédicas. Esta producción es, asimismo, mayoritariamente proveniente de colaboraciones de carácter internacional. La producción académica y su impacto en la WoS son dependientes del área científica, como ya lo habían apreciado investigaciones previas (Dorta y Dorta, 2014).

Diversos autores han destacado previamente la relación entre el número de citas y el número de colaboradores, al tiempo que han puesto en evidencia la dificultad de evaluar el grado de participación de un científico o una institución en particular en proyectos en que colaboran decenas de investigadores (Dorta y Dorta, 2010). De manera significativa, la calidad de la producción científica se ha elevado como consecuencia de la colaboración de instituciones del estado de Puebla en proyectos internacionales, como los del CERN (ALICE, CMS), la Colaboración Pierre Auger, los proyectos del Gran Telescopio Milimétrico, el Herschel-ATLAS o los del consorcio SHADES.

No es posible sobrevalorar los logros que resultan de esos grandes proyectos que no sólo amplían nuestro conocimiento científico sino que generan desarrollos en instrumentación, ingeniería y tecnología. Además de que, por la naturaleza misma de los diversos problemas de investigación, resulta necesario integrar investigadores de diferentes disciplinas, instituciones y países (Bornmann y Leydesdorf, 2015). Esta colaboración internacional que hoy, en el estado de Puebla, es creciente en las áreas de física, astronomía y astrofísica, deberá desarrollarse en las áreas científicas de la psicología, la sociología o la economía, por citar sólo algunas.

La aplicación de metodologías mixtas de manera cada vez más sólida y generalizada es otra de las causas que genera la necesidad de formar equipos de investigación integrados por especialistas de diversas áreas académicas (Creswell, 2015). Esas metodologías requieren de análisis de datos (cuantitativos y cualitativos), que se basan en modelos matemáticos y programas de cómputo cada día más complejos.

Mención aparte merece una consideración sobre la autoría de los trabajos aquí comentados: las publicaciones más citadas presentan al menos tres autores, mientras que los textos de responsabilidad individual alcanzan un máximo de seis citas en el periodo elegido para el retrato de la investigación que hemos realizado. Advirtamos, además, que todos los escritos se redactan en inglés.

Una última nota para el lector: debe destacar el hecho de que, la relevancia que en la comunidad científica se ha otorgado a la información registrada 
en la WoS, constituye sólo uno de los posibles criterios para evaluar la cantidad y calidad de la producción científica de un investigador, una institución, una región, un país. Es por demás evidente que hay más investigación en Puebla que sería puesta de manifiesto con otros criterios de evaluación de su impacto y con el análisis de otros sistemas de indexación con reconocimiento internacional.

\section{Agradecimientos}

Este trabajo ha sido parcialmente subvencionado por la Facultad de Psicología de la UAP y, en particular, A. Díaz-Furlong agradece el apoyo recibido por el Sistema Nacional de Investigadores y el CONACYT.

\section{REFERENCIAS}

Aamodt, K. et al.-ALICE collaboration. 2011a. Higher harmonic anisotropic flow measurements of charged particles in $\mathrm{Pb}-\mathrm{Pb}$ collisions at $\sqrt{s_{N N}}=2.76 \mathrm{TeV}$, Physical Review Letters, 107(3), 032301(10). DOI: <<10.1103/PhysRevLett.107.032301

Aamodt, K. et al.-ALICE collaboration-2011b. "Strange particle production in proton-proton collisions at $\sqrt{s}=0.9 \mathrm{TeV}$ with ALICE at the LHC", European Physical Journal C, vol. 71: 1594. DOI: <10.1140/epjc/s10052-011-1594-5>.

Aamodt, K. et al.-ALICE collaboration. 2010a. "Charged-particle multiplicity measurement in proton-proton collisions at $\sqrt{s}=0.9$ and $2.36 \mathrm{TeV}$ with ALICE at LHC", European Physical Journal C, vol. 68: 89-108. DOI: <10.1140/epjc/s10052-010-1339-x>.

Aamodt, K. et al.-ALICE collaboration. 2010b. "Charged-Particle Multiplicity Density at Midrapidity in Central Pb-Pb Collisions at $\sqrt{s_{N N}}=2.76 \mathrm{TeV}$ ”, Physical Review Letters, vol. 105, no. 25: 252301(11). DOI: <10.1103/PhysRevLett.105.252301>.

Aamodt, K. et al.-ALICE collaboration. 2010c. "Charged-particle multiplicity measurement in proton-proton collisions at $\sqrt{s}=7 \mathrm{TeV}$ with ALICE at LHC", European Physical Journal C, vol. 68: 345-354. DOI: <10.1140/epjc/s10052-010-1350-2>.

Aamodt, K. et al.-ALICE collaboration. 2010d. "Elliptic flow of charged particles in $\mathrm{Pb}-\mathrm{Pb}$ collisions at $\sqrt{s_{N N}}=2.76 \mathrm{TeV}$ ", Physical Review Letters, vol. 105, no. 25: 252302(11). DOI: $<<10.1103 /$ PhysRevLett.105.252302>.

Aamodt, K. et al.-ALICE collaboration. 2010e. "First proton-proton collisions at the LHC as observed with the ALICE detector: measurement of the charged-particle pseudorapidity density at $\sqrt{s}=900 \mathrm{GeV}$ ", European Physical Journal C, vol. 65: 111-125. DOI: $<10.1140 /$ epjc/s10052-009-1227-4>.

Abraham, J. et al.-The Pierre Auger Collaboration. 2008a- "Correlation of the highest-energy cosmic rays with the positions of nearby active galactic nuclei", Astroparticle Physics, vol. 29: 188-204. DOI: <10.1016/j.astropartphys.2008.01.002>. 
Abraham, J. et al.-The Pierre Auger Collaboration. 2008b. "Observation of the suppression of the flux of cosmic rays above $4 \times 10^{19} \mathrm{eV}$ ", Physical Review Letters, vol. 101, no 6: 061101(7). DOI: <10.1103/PhysRevLett.101.061101>.

Abraham, J. et al.-The Pierre Auger Collaboration. 2008c. "Upper limit on the cosmic-ray photon flux above $10^{19} \mathrm{eV}$ using the surface detector of the Pierre Auger Observatory", Astroparticle Physics, vol. 29: 243-256. DOI: <10.1016/j.astropartphys.2008.01.003>.

Abraham, J. et al.-The Pierre Auger Collaboration. 2008d. "Upper limit on the diffuse flux of ultrahigh energy tau neutrinos from the Pierre Auger Observatory", Physical Review Letters, vol. 100, no. 21: 211101(7). DOI: <10.1103/PhysRevLett.100.211101>.

Abraham, J. et al.-The Pierre Auger Collaboration. 2010a. "Measurement of the depth of maximum of extensive air showers above $10^{18} \mathrm{eV}$ ", Physical Review Letters, vol. 104, no. 9: 091101(7). DOI: <10.1103/PhysRevLett.104.091101>.

Abraham, J. et al.-The Pierre Auger Collaboration. 2010b. "Measurement of the energy spectrum of cosmic rays above $10^{18} \mathrm{eV}$ using the Pierre Auger Observatory", Physics Letters B, vol. 685: 239-246. DOI: <10.1016/j.physletb.2010.02.013>.

Abraham, J. et al.-The Pierre Auger Collaboration- 2010c. "The fluorescence detector of the Pierre Auger Observatory”, Nuclear Instruments \& Methods in Physics Research Section A - Accelerators Spectrometers Detectors and Associated Equipment, vol. 620: 227-251. DOI: <10.1016/j.nima.2010.04.023>.

Abraham, J. et al.-The Pierre Auger Collaboration. 2009a. "Limit on the diffuse flux of ultrahigh energy tau neutrinos with the surface detector of the Pierre Auger Observatory", Physical Review D, 79(10), 102001(15). DOI: <10.1103/PhysRevD.79.102001>.

Abraham, J. et al.-The Pierre Auger Collaboration. 2009b. "Upper limit on the cosmic-ray photon fraction at EeV energies from the Pierre Auger Observatory", Astroparticle Physics, vol. 31: 399-406. DOI: <10.1016/j.astropartphys.2009.04.003>.

Abreu, P. et al.-The Pierre Auger Collaboration. 2010. "Update on the correlation of the highest energy cosmic rays with nearby extragalactic matter", Astroparticle Physics, vol. 34: 314-326. DOI: <10.1016/j.astropartphys.2010.08.010>.

ALICE collaboration. 2011a. "Centrality dependence of the charged-particle multiplicity density at midrapidity in $\mathrm{Pb}-\mathrm{Pb}$ collisions at $\sqrt{s_{N N}}=2.76 \mathrm{TeV}$ ”, Physical Review Letters, vol. 106: 3: 032301(10). DOI: <10.1103/PhysRevLett.106.032301>.

ALICE collaboration. 2011b. "Suppression of charged particle production at large transverse momentum in central Pb-Pb collisions at $\sqrt{s_{N N}}=2.76 \mathrm{TeV}$ ", Physics Letters B, vol. 696:30-39. DOI: <10.1016/j.physletb.2010.12.020>.

ALICE collaboration. 2011c. "Two-pion Bose-Einstein correlations in central Pb-Pb collisions at $\sqrt{s_{N N}}=2.76 \mathrm{TeV}$ ", Physics Letters B, vol. 696: 328-337. DOI: $<10.1016 / \mathrm{j}$. physletb.2010.12.053>. 
ALICE collaboration. 2010a. "Alignment of the ALICE Inner Tracking System with cosmic-ray tracks", Journal of Instrumentation, vol. 5: P03003. DOI: $<10.1088 / 1748-0221 / 5 / 03 / \mathrm{P} 03003>$.

ALICE collaboration. 2010b. "Transverse momentum spectra of charged particles in proton-proton collisions at $\sqrt{s}=900 \mathrm{GeV}$ with ALICE at the LHC", Physics Letters $B$, vol. 693: 53-68. DOI: <10.1016/j.physletb.2010.08.026>.

ALICE collaboration. 2008. "The ALICE experiment at the CERN LHC”, Journal of Instrumentation, vol. 3: S08002. DOI: <10.1088/1748-0221/3/08/S08002>.

Allekotte, I. et al.-The Pierre Auger Collaboration. 2008. "The surface detector system of the Pierre Auger Observatory", Nuclear Instruments \& Methods in Physics Research Section A - Accelerators Spectrometers Detectors and Associated Equipment, vol. 586: 409-420. DOI: <10.1016/j.nima.2007.12.016>.

Alquicer, G. et al. 2008. "Postweaning social isolation enhances morphological changes in the neonatal ventral hippocampal lesion rat model of psychosis", Journal of Chemical Neuroanatomy, vol. 35: 179-187. DOI: <10.1016/j.jchemneu.2007.10.001>.

Althouse, B.M., J.D. West, C.T. Bergstrom y T. Bergstrom. 2009. "Differences in impact factor across fields and over time", Journal of the American Society for Information Science and Technology, vol. 60, no. 1:27-34. DOI: <10.1002/asi.20936>.

Amblard, A. et al. 2010. "Herschel-ATLAS: Dust temperature and redshift distribution of SPIRE and PACS detected sources using submillimetre colours", Astronomy \& Astrophysics, vol. 518: L9. DOI: <10.1051/0004-6361/201014586>.

Austermann, J.E. et al. 2010. "AZTEC half square degree survey of the SHADES fields I. Maps, catalogues and source counts", Monthly Notices of the Royal Astronomical Society, vol. 401: 160-176. DOI: <10.1111/j.1365-2966.2009.15620.x>.

Austermann, J.E. et al. 2009. "AZTEC Millimetre Survey of the cosmos field - II. Source count overdensity and correlations with large-scale structure", Monthly Notices of the Royal Astronomical Society, vol. 393: 1573-1583. DOI: $<10.1111 / \mathrm{j} .1365-2966.2008 .14284 . x>$.

Basilakos, S. et al. 2009. "Hubble expansion and structure formation in time varying vacuum models”, Physical Review D, vol. 80, no. 8: 083511(19). DOI: <10.1103/ PhysRevD.80.083511>.

Bisgaard, H. et al. 2008. "Study of montelukast for the treatment of respiratory symptoms of post-respiratory syncytial virus bronchiolitis in children”, American Journal of Respiratory and Critical Care Medicine, vol. 178: 854-860. DOI: <10.1164/ rccm.200706-910OC>.

Bornmann, L. y L. Leydesdorf. 2015. “Topical connections between the institutions within an organisation (institutional co-authorships, direct citation links and co-citations)", Scientometrics, vol. 102: 455-463. DOI: <10.1007/s11192-0141425-1>. 
Bosman, M. y T.R. Anoro. 2012. "La búsqueda del bosón de Higgs”, Investigación y Ciencia, vol. 432: 16-23.

Chapin, E.L. et al. 2009. "An AZTEC 1.1 mm survey of the GOODS-N field - II. Multiwavelength identifications and redshift distribution, Monthly Notices of the Royal Astronomical Society, vol. 398: 1793-1808. DOI: <10.1111/j.1365-2966.2009.15267.x>.

Chapin, E.L. et al. 2008. "The Balloon-borne Large Aperture Submillimeter Telescope (BLAST) 2005: A $4 \mathrm{deg}^{2}$ Galactic plane survey in Vulpecula $\left(l=59^{\circ}\right)$ ", Astrophysical Journal, vol. 681: 428-452. DOI: <10.1086/588544>.

Chatrchyan, S. et al.- CMS collaboration. 2012. "Search for the Standard Model Higgs Boson in the Decay Channel $H \rightarrow Z Z \rightarrow 4 l$ in $p p$ Collisions at $\sqrt{s}=7 \mathrm{TeV}$ ", Physical Review Letters, vol. 108, no. 11: 111804(17). DOI: <10.1103/PhysRevLett.108.111804>.

Chatrchyan, S. et al.- CMS collaboration. 2011a. "Indications of suppression of excited upsilon states in $\mathrm{Pb}-\mathrm{Pb}$ Collisions at $\sqrt{s_{N N}}=2.76 \mathrm{TeV}$ ”, Physical Review Letters, vol. 107, no. 5: 052302(15). DOI: <10.1103/PhysRevLett.107.052302>.

Chatrchyan, S. et al. -CMS collaboration. 2011b. "Observation and studies of jet quenching in $\mathrm{PbPb}$ collisions at $\sqrt{s_{N N}}=2.76 \mathrm{TeV}$ ", Physical Review C, vol. 84, no. 2: 024906(26). DOI: <10.1103/PhysRevC.84.024906>.

Chatrchyan, S. et al. -CMS collaboration. 2011c. "Search for neutral minimal supersymmetric standard model Higgs bosons decaying to tau pairs in $p p$ collisions at $\sqrt{s}=7$ TeV", Physical Review Letter, vol. 106, no. 23: 231801(15). DOI: <10.1103/ PhysRevLett.106.231801>.

Clements, D.L. et al. 2008. “The SCUBA HAlf-Degree Extragalactic Survey (SHADES) - VIII. The nature of faint submillimetre galaxies in SHADES, SWIRE and SXDF surveys", Monthly Notices of the Royal Astronomical Society, vol. 387: 247-267. DOI: $<10.1111 / \mathrm{j} .1365-2966.2008 .13172 . \mathrm{x}>$.

CMS collaboration. 2012a. "Combined results of searches for the standard model Higgs boson in $p p$ collisions at $\sqrt{s}=7 \mathrm{TeV}$ ", Physics Letters B, vol. 710: 26-48. DOI: $<10.1016 /$ j.physletb.2012.02.064>.

CMS collaboration. 2012b. "Observation of a new boson at a mass of $125 \mathrm{GeV}$ with the CMS experiment at the LHC", Physics Letters B, vol. 716: 30-61. DOI: $<10.1016 /$ j.physletb.2012.08.021>.

CMS collaboration. 2012c. "Search for neutral Higgs bosons decaying to tau pairs in pp collisions at $\sqrt{s}=7$ TeV", Physics Letters B, vol. 713: 68-90. DOI: $<10.1016 / \mathrm{j}$. physletb.2012.05.028>.

CMS collaboration. 2012d. "Search for the standard model Higgs boson decaying into two photons in $p p$ collisions at $\sqrt{s}=7 \mathrm{TeV}$ ", Physics Letters B, vol. 710: 403-425. DOI: $<10.1016 /$ j.physletb.2012.03.003>.

CMS collaboration. 2012e. "Search for the standard model Higgs boson decaying to $\mathrm{W}+\mathrm{W}$ - in the fully leptonic final state in $p p$ collisions at $\sqrt{s}=7 \mathrm{TeV}$ ", Physics Letters B, vol. 710: 91-113. DOI: <10.1016/j.physletb.2012.02.076>. 
CMS collaboration. 2011a. "Determination of jet energy calibration and transverse momentum resolution in CMS", Journal of Instrumentation, vol. 6, P11002. DOI: $<10.1088 / 1748-0221 / 6 / 11 /$ P11002>.

CMS collaboration. 2011b. "First measurement of the cross section for top-quark pair production in proton-proton collisions at $\sqrt{s}=7 \mathrm{TeV}$ ", Physics Letters B, vol. 695: 424-443. DOI: <10.1016/j.physletb.2010.11.058>.

CMS collaboration. 2011c. "Measurements of inclusive $\mathrm{W}$ and $\mathrm{Z}$ cross sections in $p p$ collisions at $\sqrt{s}=7 \mathrm{TeV}$ ", Journal of High Energy Physics, vol. 1: 1-40. DOI: $<10.1007 /$ JHEP01(2011)080>.

CMS collaboration. 2011d. "Measurement of W+W- production and search for the Higgs boson in $p p$ collisions at $\sqrt{s}=7 \mathrm{TeV}$ ", Physics Letters B, vol. 699: 25-47. DOI: $<10.1016 /$ j.physletb.2011.03.056>.

CMS collaboration. 2011f. "Prompt and non-prompt J/psi production in $p p$ collisions at $\sqrt{s}=7 \mathrm{TeV}$ ", European Physical Journal C, vol. 71, no. 1575. DOI: <10.1140/epjc/ s10052-011-1575-8>.

CMS collaboration. 2011g. "Search for resonances in the dijet mass spectrum from 7 TeV pp collisions at CMS”, Physics Letters B, vol. 704: 123-142. DOI: <10.1016/j. physletb.2011.09.015>.

CMS collaboration. 2011h. "Search for supersymmetry at the LHC in events with jets and missing transverse energy", Physical Review Letters, vol. 107, no. 22: 221804(16). DOI: <10.1103/PhysRevLett.107.221804>.

CMS collaboration. 2011i. "Search for supersymmetry in $p p$ collisions at $7 \mathrm{TeV}$ in events with jets and missing transverse energy", Physics Letters B, vol. 698: 196218. DOI: <10.1016/j.physletb.2011.03.021>.

CMS collaboration. 2010a. "Observation of long-range, near-side angular correlations in proton-proton collisions at the LHC”, Journal of High Energy Physics, vol. 9, no. 91. DOI: <10.1007/JHEP09(2010)091>.

CMS collaboration. 2010b. "Search for dijet resonances in $7 \mathrm{TeV} p p$ collisions at CMS", Physical Review Letters, vol. 105, no. 21: 211801 (14). DOI: <10.1103/PhysRevLett.105.211801>.

CMS collaboration. 2010c. "Transverse-momentum and pseudorapidity distributions of charged hadrons in $p p$ collisions at $\sqrt{s}=0.9$ and $2.36 \mathrm{TeV}$ ", Journal of High Energy Physics, vol. 2, no. 41. DOI: <10.1007/JHEP02(2010)041>.

Colberg, J.M. et al. 2008. "The Aspen-Amsterdam void finder comparison project", Montbly Notices of the Royal Astronomical Society, vol. 387: 933-944. DOI: $<10.1111 / \mathrm{j} .1365-2966.2008 .13307 . x>$.

Coppin, K. et al. 2008. "The SCUBA HAlf degree extragalactic survey - VI. 350- $\mu \mathrm{m}$ mapping of submillimetre galaxies”, Monthly Notices of the Royal Astronomical Society, vol. 384, 1597-1610. DOI: <10.1111/j.1365-2966.2007.12808.x>. 
CARACTERÍSTICAS DE LA PRODUCCIÓN CIENTÍFICA DE INSTITUCIONES DE PUEBLA...

Creswell, J.W. 2015. A Concise Introduction to Mixed Methods Research, Londres: Sage.

Das, S. et al. 2011. "The Atacama cosmology telescope: A measurement of the cosmic microwave background power spectrum at 148 and $218 \mathrm{GHz}$ from the 2008 southern survey", Astrophysical Journal, vol. 729, no. 62. DOI: <10.1088/0004637X/729/1/62>.

Devlin, M.J. et al. 2009. "Over half of the far-infrared background light comes from galaxies at $z \geqq 1.2$ ”, Nature, no. 458: 737-739. DOI: <10.1038/nature07918>.

Dorta González, M.I. y P. Dorta González. 2014. "Factor de impacto agregado según campos científicos”, Investigación Bibliotecológica, vol. 28:15-28.

Dorta González, M.I. y P. Dorta González. 2010. "Indicador bibliométrico basado en el índice b”, Revista Española de Documentación Cientifica, vol. 33: 225-245. DOI: $<10.3989 /$ redc. $2010.2 .733>$.

Dunkley, J. et al. 2011. "The Atacama cosmology telescope: cosmological parameters from the 2008 power spectra”, Astrophysical Journal, vol. 739: 1-20. DOI: $<10.1088 / 0004-637 X / 739 / 1 / 52>$.

Dye, S. et al. 2009. "Radio and mid-infrared identification of BLAST source counterparts in the Chandra Deep Field South”, Astrophysical Journal, vol. 703: 285-299. DOI: $<10.1088 / 0004-637 \mathrm{X} / 703 / 1 / 285>$.

Dye, S. et al. 2008. "The SCUBA HAlf degree extragalactic survey (SHADES) - VII. Optical/IR photometry and stellar masses of submillimetre galaxies", Monthly Notices of the Royal Astronomical Society, vol. 386: 1107-1130. DOI: $<10.1111 / \mathrm{j} .1365-2966.2008 .13113 . \mathrm{x}>$.

Eales, S. et al. 2010. "The Herschel ATLAs", Publications of the Astronomical Society of the Pacific, vol. 122: 499-515.

Eaves-Pyles, T. et al. 2008. "Escherichia coli isolated from a Crohn's disease patient adheres, invades, and induces inflammatory responses in polarized intestinal epithelial cells", International Journal of Medical Microbiology, vol. 298: 397-409. DOI: <10.1016/j.ijmm.2007.05.011>.

Fowler, J.W. et al. 2010. "The Atacama cosmology telescope: A measurement of the $600<l<8000$ cosmic microwave background power spectrum at $148 \mathrm{GHz}$, Astrophysical Journal, vol. 722: 1148-1161. DOI: <10.1088/0004-637X/722/2/ $1148>$.

Ganapathy, R. et al. 2008. "Soliton interaction under soliton dispersion management", IEEE Journal of Quantum Electronics, 44: 383-390. DOI: <10.1109/ JQE.2007.914778>.

García-Carrasco, M. et al. 2009. "Use of rituximab in patients with systemic lupus erythematosus: An update”, Autoimmunity Reviews, vol. 8: 343-348. DOI: $<10.1016 /$ j.autrev.2008.11.006>. 
Gómez-Almaguer, D. et al. 2008. "Alemtuzumab for the treatment of steroid-refractory acute graft-versus-host disease", Biology of Blood and Marrow Transplantation, vol. 14: 10-15. doi: <10.1016/j.bbmt.2007.08.052>.

Gutermuth, R.A. et al. 2008. "Spitzer observations of NGC 1333: A study of structure and evolution in a nearby embedded cluster", Astrophysical Journal, vol. 674:336356. DOI: $<10.1086 / 524722>$.

Hägele, G.F. et al. 2008. "Precision abundance analysis of bright HII galaxies", Monthly Notices of the Royal Astronomical Society, vol. 383: 209-229. DOI: <10.1111/ j.1365-2966.2007.12527.x>.

Hickmann, J.M. et al. 2010. "Unveiling a Truncated Optical Lattice Associated with a Triangular Aperture Using Light's Orbital Angular Momentum”, Physical Review Letters, vol. 105, no. 5: 053904(4). DOI: <10.1103/PhysRevLett.105.053904>.

Hincks, A.D. et al. 2010. "The Atacama Cosmology Telescope (ACT): Beam profiles and First SZ cluster maps", Astrophysical Journal Supplement Series, vol. 191: 423-438. DOI: <10.1088/0067-0049/191/2/423>.

Hirsch, J.E. 2005. "An index to quantify an individual's scientific research output", Proceedings of the National Academy of Sciences of the United States of America, vol. 102: 16569-16572. DOI: <10.1073/pnas.0507655102>.

Iocco, F. et al. 2008. "Dark matter annihilation effects on the first stars", Monthly Notices of the Royal Astronomical Society, 390: 1655-1669. DOI: $<10.1111 /$ j.1365-2966.2008.13853.x $>$.

Ivison, R.J. et al. 2010. "BLAST: The far-infrared/radio correlation in distant galaxies", Monthly Notices of the Royal Astronomical Society, vol. 402: 245-258. DOI: $<10.1111 / j .1365-2966.2009 .15918 . x>$.

Juárez, Y. et al. 2009. "The metallicity of the most distant quasars", Astronomy $\&$ Astrophysics, vol. 494: L25-L28. DOI: <10.1051/0004-6361:200811415>.

Kartashov, Y.V., B.A. Malomed, V.A. Vysloukh y L. Torner. 2009a. "Two-dimensional solitons in nonlinear lattices”, Optics Letters, vol. 34: 770-772.

Kartashov, Y.V., V.A. Vysloukh y L. Torner. 2009b. "Soliton shape and mobility control in optical lattices", Progress in Optics, vol. 52: 63-148. DOI: <10.1016/S00796638(08)00004-8>.

Khachatryan V. et al.-CMS collaboration. 2010. "Transverse-momentum and pseudorapidity distributions of charged hadrons in $p p$ collisions at $\sqrt{s}=7 \mathrm{TeV}$ ", Physical Review Letters, vol. 105, no. 2: 022002(14). DOI: <10.1103/PhysRevLett.105.022002>.

Kuhl, U. et al. 2008. "Enhancement of localization in one-dimensional random potentials with long-range correlations", Physical Review Letters, vol. 100, no. 12: 126402(4). DOI: <10.1103/PhysRevLett.100.126402>.

Lara, C. et al. 2009. "Childhood predictors of adult attention-deficit/hyperactivity disorder: Results from the World Health Organization World Mental Health 
CARACTERÍSTICAS DE LA PRODUCCIÓN CIENTÍFICA DE INSTITUCIONES DE PUEBLA...

Survey Initiative”, Biological Psychiatry, vol. 65: 46-54. DOI: <10.1016/j. biopsych.2008.10.005>.

Liu, H.B et al. 2008. "Thermodynamic stability and melting mechanism of bimetallic Au-Pt nanoparticles”, Journal of Physical Chemistry C, vol. 112: 19173-19177. DOI: $<10.1021 /$ jp802804u $>$.

Marigo, P. et al. 2008. "Evolution of asymptotic giant branch stars - II. Optical to far-infrared isochrones with improved TP-AGB models", Astronomy \& Astrophysics, vol. 482: 883-905. DOI: <10.1051/0004-6361:20078467>.

Marriage, T.A. et al. 2011. “The Atacama Cosmology Telescope: Sunyaev-Zel'dovich-selected galaxy clusters at $148 \mathrm{GHz}$ in the 2008 survey, Astrophysical Journal, vol. 737, no. 61. DOI: <10.1088/0004-637X/737/2/61>.

Marsden, G. et al. 2009. "BLAST: Resolving the cosmic submillimeter background", Astrophysical Journal, vol. 707: 1729-1739. DOI: <10.1088/0004637X/707/2/1729>.

Martínez Huitle, C.A. et al. 2008. "Removal of the pesticide methamidophos from aqueous solutions by electrooxidation using $\mathrm{Pb} / \mathrm{PbO}_{2}, \mathrm{Ti} / \mathrm{SnO}_{2}$, and $\mathrm{Si} / \mathrm{BDD}$ electrodes", Environmental Science \& Technology, vol. 42: 6929-6935. DOI: $<10.1021$ /es8008419>.

Martínez Téllez, R.I. et al. 2009. "Prenatal stress alters spine density and dendritic length of nucleus accumbens and hippocampus neurons in rat offspring”, Synapse, vol. 63: 794-804. DOI: <10.1002/syn.20664>.

Miller, P.J. et al. 2010. "Newcastle disease: Evolution of genotypes and the related diagnostic challenges", Infection Genetics and Evolution, vol. 10: 26-35. DOI: $<10.1016 /$ j.meegid.2009.09.012>.

Negrello, M. et al. 2010. "The detection of a population of submillimeter-bright, strongly lensed galaxies", Science, no. 330: 800-804. DOI: <10.1126/science.1193420>.

Netterfield, C.B. et al. 2009. "BLAST: The mass function, lifetimes, and properties of intermediate mass cores from a 50 square degree submillimeter galactic survey in Vela $\left(l \approx 265^{\circ}\right)$ ", Astrophysical Journal, vol. 707: 1824-1835. DOI: $<10.1088 / 0004$ $637 \mathrm{X} / 707 / 2 / 1824>$.

Pascale, E. et al. 2008. “The Balloon-borne Large Aperture Submillimeter Telescope: BLAST”, Astrophysical Journal, vol. 681: 400-414. DOI: <10.1086/588541>.

Pascale, E. et al. 2009. "BLAST: A far-infrared measurement of the history of star formation”, Astropbysical Journal, vol. 707: 1740-1749. DOI: <10.1088/0004637X/707/2/1740>.

Patanchon, G. et al. 2008. "Sanepic: A mapmaking method for time stream data from large arrays”, Astrophysical Journal, vol. 681: 708-725. DOI: $<10.1086 / 588543>$. 
Patanchon, G. et al. 2009. "Submillimeter number counts from statistical analysis of blast maps", Astrophysical Journal, vol. 707: 1750-1765. doi: <10.1088/0004637X/707/2/1750>.

Perera, T.A. et al. 2008. "An AZTEC $1.1 \mathrm{~mm}$ survey of the goods-N field - I. Maps, catalogue and source statistics", Monthly Notices of the Royal Astronomical Society, vol. 391: 1227-1238. DOI: $<10.1111 /$ j.1365-2966.2008.13902.x>.

Real Academia Española. 2014. Diccionario de la lengua española, $23^{a}$ ed. Madrid: Espasa Calpe.

Ramírez San-Juan, J.C. et al. 2008. "Impact of velocity distribution assumption on simplified laser speckle imaging equation", Optics Express, vol. 16: 3197-3203. DOI: $<10.1364 / \mathrm{OE} .16 .003197$

Rodríguez Zurita, G. et al. 2008. "One-shot phase-shifting phase-grating interferometry with modulation of polarization: case of four interferograms", Optics Express, vol. 16, 7806-7817. DOI: <10.1364/OE.16.007806>.

Saldaña, Z. et al. 2009. "Synergistic role of curli and cellulose in cell adherence and biofilm formation of attaching and effacing Escherichia coli and identification of Fis as a negative regulator of curli", Environmental Microbiology, vol. 11: 9921006. DOI: <10.1111/j.1462-2920.2008.01824.x>.

Scior, T. et al. 2009. "How to recognize and workaround pitfalls in QSAR studies: A critical review", Current Medicinal Chemistry, vol. 16: 4297-4313.

Scott, K.S. et al. 2008. "AZTEC millimetre survey of the cosmos field - I. Data reduction and source catalogue", Monthly Notices of the Royal Astronomical Society, vol. 385: 2225-2238. DOI: <10.1111/j.1365-2966.2008.12989.x>.

Scott, K.S. et al. 2010. "Deep $1.1 \mathrm{~mm}$-wavelength imaging of the GOODS-s field by AZTEC /ASTE - I. Source catalogue and number counts", Monthly Notices of the Royal Astronomical Society, vol. 405: 2260-2278. DOI: <10.1111/j.1365-2966.2010.16644.x>.

Sehgal, N. et al. 2011. "The Atacama Cosmology Telescope: Cosmology from galaxy clusters detected via the Sunyaev-Zel'dovich effect", Astrophysical Journal, vol. 732, no. 44. DOI: <10.1088/0004-637X/732/1/44>.

Serkin, V.N. et al. 2010. "Nonautonomous matter-wave solitons near the Feshbach resonance", Physical Review A, vol. 81, no. 2: 023610(19). DOI: <10.1103/PhysRevA.81.023610>.

Szameit, A. et al. 2009. "Inhibition of light tunneling in waveguide arrays", Physical Review Letters, vol. 102, no. 15: 153901(4). DOI: <10.1103/PhysRevLett.102.153901>.

Testa, J. 2003. "The Thomson ISI Journal selection process", Serials Review, vol. 29, no. 3: 210-212. DOI: <10.1016/S0098-7913(03)00063-7>.

Vega, O. et al. 2008. "Modelling the spectral energy distribution of ULIRGS - II. The energetic environment and the dense interstellar medium", Astronomy \& Astrophysics, vol. 484, no. 631: U29. DOI: <10.1051/0004-6361:20078883>. 
Viero, M.P. et al. 2009. "BLAST: Correlations in the cosmic far-infrared background at 250,350 , and $500 \mu \mathrm{m}$ reveal clustering of star-forming galaxies", Astrophysical Journal, vol. 707: 1766-1778. DOI: <10.1088/0004-637X/707/2/1766>.

\section{Para citar este texto:}

Díaz-Cárdenas, Alfonso Felipe; Sankey-García, María del Rayo; Díaz-Furlong, Alfonso; Díaz-Furlong, Héctor Adrián; Xoxocotzi-Aguilar, Reyna; Vázquez-López, José Jaime; Apam-García, Cynthia. 2017. "Características de la producción científica de instituciones de Puebla indexada en la Web of Science". Investigación Bibliotecológica: Archivonomia, Bibliotecología e Información (Número Especial de Bibliometría): 43-77.

http://dx.doi.org/10.22201/iibi.24488321xe.2017.nesp1.57885 